\title{
Scalar curvature for noncommutative four-tori
}

\author{
Farzad Fathizadeh and Masoud Khalkhali
}

\begin{abstract}
In this paper we study the curved geometry of noncommutative 4-tori $\mathbb{T}_{\theta}^{4}$. We use a Weyl conformal factor to perturb the standard volume form and obtain the Laplacian that encodes the local geometric information. We use Connes' pseudodifferential calculus to explicitly compute the terms in the small time heat kernel expansion of the perturbed Laplacian which correspond to the volume and scalar curvature of $\mathbb{T}_{\theta}^{4}$. We establish the analogue of Weyl's law, define a noncommutative residue, prove the analogue of Connes' trace theorem, and find explicit formulas for the local functions that describe the scalar curvature of $\mathbb{T}_{\theta}^{4}$. We also study the analogue of the Einstein-Hilbert action for these spaces and show that metrics with constant scalar curvature are critical for this action.
\end{abstract}

Mathematics Subject Classification (2010). 46L87, 58B34, 58J42.

Keywords. Noncommutative geometry, scalar curvature, Einstein-Hilbert action, Weyl's law, noncommutative residue, Connes' trace theorem, heat kernel, noncommutative four-tori.

\section{Contents}

1 Introduction . . . . . . . . . . . . . . . . . . . . . 473

2 Noncommutative tori . . . . . . . . . . . . . . . . . 475

3 Laplacian and its heat kernel . . . . . . . . . . . . . . . . . 478

4 Weyl's law and Connes' trace theorem . . . . . . . . . . . . . . . 483

5 Scalar curvature and Einstein-Hilbert action . . . . . . . . . . . . . . 491

References . . . . . . . . . . . . . . . . . . . . 502

\section{Introduction}

Spectral geometry has played an important role in the development of metric aspects of noncommutative geometry $[6,8,10,9]$. After the seminal paper [12], in which the analogue of the Gauss-Bonnet theorem is proved for noncommutative two tori $\mathbb{T}_{\theta}^{2}$, there has been much progress in understanding the local differential geometry of these noncommutative spaces $[15,11,16,17]$. In these works, the flat geometry 
of $\mathbb{T}_{\theta}^{2}$ which was studied in [5] is conformally perturbed by means of a Weyl factor given by a positive invertible element in $C^{\infty}\left(\mathbb{T}_{\theta}^{2}\right)$ (see also [4] for a preliminary version). The pseudodifferential calculus developed in [5] for $C^{*}$-dynamical systems is employed crucially to apply heat kernel techniques to geometric operators on $\mathbb{T}_{\theta}^{2}$ to derive small time heat kernel expansions that encode local geometric information such as scalar curvature. A purely noncommutative feature is the appearance of the modular automorphism of the state implementing the conformal perturbation of the metric in the computations and in the final formula for the curvature $[11,16]$.

In this paper we study the curved geometry of noncommutative 4-tori $\mathbb{T}_{\theta}^{4}$. We view these spaces as noncommutative abelian varieties equipped with a complex structure given by the simplest possible period matrix. We use a Weyl conformal factor to perturb the standard volume form and obtain the Laplacian that encodes the local geometric information. We use the pseudodifferential calculus of [5] to explicitly compute the terms in the small time heat kernel expansion of the perturbed Laplacian which correspond to the volume and scalar curvature of $\mathbb{T}_{\theta}^{4}$. We establish the analogue of Weyl's law, define a noncommutative residue, prove the analogue of Connes' trace theorem [7], and find explicit formulas for the local functions that describe the scalar curvature of $\mathbb{T}_{\theta}^{4}$. We also study the analogue of the EinsteinHilbert action for these spaces and show that metrics with constant scalar curvature are critical for this action.

This paper is organized as follows. In Section 2, we recall basic facts about higher dimensional noncommutative tori and their flat geometry. In Section 3, we consider the noncommutative 4-torus $\mathbb{T}_{\theta}^{4}$ with the simplest structure of a noncommutative abelian variety. We perturb the standard volume form on this space conformally (cf. [12]), and analyse the corresponding perturbed Laplacian. Then, we recall the pseudodifferential calculus of [5] for $\mathbb{T}_{\theta}^{4}$ and review the derivation of the small time heat kernel expansion for the perturbed Laplacian, using this calculus. In Section 4, we prove the analogue of Weyl's law for $\mathbb{T}_{\theta}^{4}$ by studying the asymptotic distribution of the eigenvalues of the perturbed Laplacian on this space. We then define a noncommutative residue on the algebra of classical pseudodifferential operators on $\mathbb{T}_{\theta}^{4}$, and show that it gives the unique continuous trace on this algebra. We also prove a trace theorem for $\mathbb{T}_{\theta}^{4}$ by showing that this noncommutative residue and the Dixmier trace coincide on pseudodifferential operators of order -4. In Section 5, we perform the computation of the scalar curvature for $\mathbb{T}_{\theta}^{4}$, and find explicit formulas for the local functions that describe the curvature in terms of the modular automorphism of the conformally perturbed volume form and derivatives of the logarithm of the Weyl factor. Then, by integrating this curvature, we define and find an explicit formula for the analogue of the Einstein-Hilbert action for $\mathbb{T}_{\theta}^{4}$. Finally, we show that the extremum of this action occurs at metrics with constant scalar curvature (see [3] for the corresponding commutative statement).

We are indebted to Alain Connes for several enlightening discussions at different stages of this work. Also, F. F. would like to thank IHES for the excellent 
environment and kind support during his visit in Winter 2012, where part of this work was carried out.

\section{Noncommutative tori}

In this section we recall basic facts about higher dimensional noncommutative tori and their flat geometry.

2.1. Noncommutative real tori. Let $V$ be a finite dimensional real vector space equipped with a positive definite inner product $\langle$,$\rangle and let \theta: V \otimes V \rightarrow \mathbb{R}$ be a skew-symmetric bilinear form on $V$. Let $\Lambda \subset V$ be a cocompact lattice in $V$. Thus $\Lambda$ is a discrete abelian subgroup of $V$ such that the quotient space $V / \Lambda$ is compact. Equivalently, we can describe $\Lambda$ as $\Lambda=\mathbb{Z} e_{1}+\cdots+\mathbb{Z} e_{n}$, the free abelian group generated by a linear basis $e_{1}, \ldots, e_{n}$ for $V$.

By definition, the noncommutative torus $C\left(\mathbb{T}_{\theta}^{n}\right)$, attached to the above data, is the universal unital $C^{*}$-algebra generated by unitaries $U_{\alpha}, \alpha \in \Lambda$, satisfying the relations

$$
U_{\alpha} U_{\beta}=e^{\pi i \theta(\alpha, \beta)} U_{\alpha+\beta}, \quad \alpha, \beta \in \Lambda .
$$

Let $e_{1}, \ldots, e_{n}$ be a basis for $\Lambda$ over $\mathbb{Z}$, and let $U_{i}=U_{e_{i}}$. Then we have

$$
U_{k} U_{l}=e^{2 \pi i \theta_{k l}} U_{l} U_{k}, \quad k, l=1, \ldots, n,
$$

where $\theta_{k l}=\theta\left(e_{k}, e_{l}\right)$.

Let $\Lambda^{\prime} \subset V$ denote the dual lattice. Thus $v \in \Lambda^{\prime}$ iff $\langle v, w\rangle \in 2 \pi \mathbb{Z}$ for all $w \in \Lambda$. There is a continuous action of the dual torus $V / \Lambda^{\prime}$ on $C\left(\mathbb{T}_{\theta}^{n}\right)$ by $C^{*}$ algebra automorphisms $\left\{\lambda_{s}\right\}_{s \in V}$, defined by

$$
\lambda_{s}\left(U_{\alpha}\right)=e^{i\langle s, \alpha\rangle} U_{\alpha}
$$

The space of smooth elements of this action, namely those elements $a \in C\left(\mathbb{T}_{\theta}^{n}\right)$ for which the map $s \rightarrow \lambda_{s}(a)$ is smooth will be denoted by $C^{\infty}\left(\mathbb{T}_{\theta}^{n}\right)$. It is a dense $*$-subalgebra of $C\left(\mathbb{T}_{\theta}^{n}\right)$ which plays the role of smooth functions on the noncommutative torus $\mathbb{T}_{\theta}^{n}$. It can be alternatively described as the algebra of elements in $C\left(\mathbb{T}_{\theta}^{n}\right)$ whose (noncommutative) Fourier expansion has rapidly decreasing Schwartz class coefficients:

$$
C^{\infty}\left(\mathbb{T}_{\theta}^{n}\right)=\left\{\sum_{\alpha \in \Lambda} a_{\alpha} U_{\alpha} ; \quad \sup _{\alpha \in \Lambda}\left(|\alpha|^{k}\left|a_{\alpha}\right|\right)<\infty, \forall k \in \mathbb{N}\right\} .
$$

There is a normalized faithful positive trace, i.e. a tracial state, $\varphi_{0}$ on $C\left(\mathbb{T}_{\theta}^{n}\right)$ whose restriction on smooth elements is given by

$$
\varphi_{0}\left(\sum_{\alpha \in \mathbb{Z}^{n}} a_{\alpha} U_{\alpha}\right)=a_{0}
$$


The infinitesimal generator of the action $\lambda_{s}$ defines a Lie algebra map

$$
\delta: V \rightarrow \operatorname{Der}\left(C^{\infty}\left(\mathbb{T}_{\theta}^{n}\right), C^{\infty}\left(\mathbb{T}_{\theta}^{n}\right)\right),
$$

where we have used the natural identification of the (abelian) Lie algebra of the torus $V / \Lambda^{\prime}$ with $V$.

Let us fix an orthonormal basis $e_{1}, \ldots, e_{n}$ for $V$. Then the restriction of the above map $\delta$ defines commuting derivations $\delta_{i}:=\delta\left(e_{i}\right): C^{\infty}\left(\mathbb{T}_{\theta}^{n}\right) \rightarrow C^{\infty}\left(\mathbb{T}_{\theta}^{n}\right)$, $i=1, \ldots, n$, which satisfy

$$
\delta_{i}\left(U_{j}\right)=\delta_{i j} U_{i}, \quad i, j=1, \ldots, n .
$$

The derivations $\delta_{j}$ are analogues of the differential operators $\frac{1}{i} \partial / \partial x_{j}$ acting on smooth functions on the ordinary torus. We have $\delta_{j}\left(a^{*}\right)=-\delta_{j}(a)^{*}$ for $j=1, \ldots, n$, and any $a \in C^{\infty}\left(\mathbb{T}_{\theta}^{n}\right)$. Moreover, since $\varphi_{0} \circ \delta_{j}=0$, for all $j$, we have the analogue of the integration by parts formula:

$$
\varphi_{0}\left(a \delta_{j}(b)\right)=-\varphi_{0}\left(\delta_{j}(a) b\right), \quad a, b \in C^{\infty}\left(\mathbb{T}_{\theta}^{n}\right) .
$$

Using these derivations, we can define the flat Laplacian

$$
\triangle=\sum_{i=1}^{n} \delta_{i}^{2}: C^{\infty}\left(\mathbb{T}_{\theta}^{n}\right) \rightarrow C^{\infty}\left(\mathbb{T}_{\theta}^{n}\right) .
$$

We note that the Laplacian $\triangle$ is independent of the choice of the orthonormal basis $e_{1}, \ldots, e_{n}$.

2.2. Noncommutative complex tori. Let $W$ be an $n$-dimensional complex vector space and $\Lambda \subset W$ be a lattice in $W$. Thus $\Lambda$ is a free abelian group of rank $2 n$ which is discrete in its subspace topology. Given a basis $e_{1}, \ldots, e_{n}$ of $W$ as a complex vector space and a basis $\lambda_{1}, \ldots, \lambda_{2 n}$ of $\Lambda$ as a free abelian group, we can express $\lambda_{1}, \ldots, \lambda_{2 n}$ in terms of $e_{1}, \ldots, e_{n}$. We obtain an $n$ by $2 n$ matrix $\mathcal{M}=(A, B)$ with $A, B \in M_{n}(\mathbb{C})$ with

$$
\lambda_{j}=\sum_{i=1}^{n} \mathcal{M}_{i j} e_{i}, \quad j=1, \ldots, 2 n .
$$

Let $W_{\mathbb{R}}$ denote the realification of $W$. Note that $\lambda_{1}, \ldots, \lambda_{2 n}$ is a basis for $W_{\mathbb{R}}$ over $\mathbb{R}$. Let $d z_{1}, \ldots, d z_{n}$ denote the basis of $W^{*}=\operatorname{Hom}_{\mathbb{C}}(W, \mathbb{C})$, dual to the basis $e_{1}, \ldots, e_{n}$, and let $d x_{1}, \ldots, d x_{2 n}$ denote the basis of $W_{\mathbb{R}}^{*}=\operatorname{Hom}_{\mathbb{R}}(W, \mathbb{R})$, dual to the basis $\lambda_{1}, \ldots, \lambda_{2 n}$. Then we have for $i=1, \ldots, n$,

$$
d z_{i}=\sum_{j=1}^{2 n} \mathcal{M}_{i j} d x_{j}, \quad d \bar{z}_{i}=\sum_{j=1}^{2 n} \overline{\mathcal{M}}_{i j} d x_{j}
$$


Now let $\theta: W_{\mathbb{R}} \otimes W_{\mathbb{R}} \rightarrow \mathbb{R}$ be an alternating bilinear form and $\mathbb{T}_{\theta}^{n}$ denote the associated noncommutative torus. We further assume that $W_{\mathbb{R}}$ is equipped with an Euclidean inner product. Using (2.1), we get the derivations

$$
\delta_{i}=\delta_{\lambda_{i}}: C^{\infty}\left(\mathbb{T}_{\theta}^{n}\right) \rightarrow C^{\infty}\left(\mathbb{T}_{\theta}^{n}\right), \quad i=1, \ldots, 2 n .
$$

The above relations define the Dolbeault operators which will be denoted by $\partial_{i}, \bar{\partial}_{i}, i=1, \ldots, n$.

We have a decomposition $W_{\mathbb{R}} \otimes \mathbb{C}=W_{(1,0)} \oplus W_{(0,1)}$ with $W_{(1,0)}=W$ and $W_{(0,1)}=\bar{W}$ with the bases $e_{1}, \ldots, e_{n}$ for $W_{(1,0)}$ and $\bar{e}_{1}, \ldots, \bar{e}_{n}$ for $W_{(0,1)}$. Let $d z_{i}$ and $d \bar{z}_{j}$ denote the corresponding bases for dual spaces $W_{(1,0)}^{*}=\operatorname{Hom}_{\mathbb{C}}\left(W_{(1,0)}, \mathbb{C}\right)$ and $W_{(0,1)}^{*}$. Using this decomposition we can define a Dolbeault type complex for on $C^{\infty}\left(\mathbb{T}_{\theta}^{n}\right)$ as follows. Let

$$
\Omega^{p, q}:=C^{\infty}\left(\mathbb{T}_{\theta}^{n}\right) \otimes \wedge^{p} W_{(1,0)}^{*} \otimes \wedge^{q} W_{(0,1)}^{*},
$$

and define the operators

$$
\partial_{i}: \Omega^{p, q} \rightarrow \Omega^{p+1, q}, \quad \bar{\partial}_{i}: \Omega^{p, q} \rightarrow \Omega^{p, q+1} .
$$

by

$$
\begin{aligned}
& \partial_{i}\left(a d z_{I} \wedge d \bar{z}_{J}\right)=\sum_{i} \partial_{i}(a) d z_{i} \wedge d z_{I} \wedge d \bar{z}_{J}, \\
& \bar{\partial}_{i}\left(a d z_{I} \wedge d \bar{z}_{J}\right)=\sum_{i} \bar{\partial}_{i}(a) d \bar{z}_{i} \wedge d z_{I} \wedge d \bar{z}_{J} .
\end{aligned}
$$

Suppressing the obvious indexing, these operators satisfy the relations

$$
\partial^{2}=0, \quad \bar{\partial}^{2}=0, \quad \partial \bar{\partial}+\bar{\partial} \partial=0 .
$$

Let $\mathfrak{H}_{n} \subset M_{n}(\mathbb{C})$ denote the Siegel upper half space. By definition, a matrix $\Omega \in \mathfrak{H}_{n}$ if and only if

$$
\Omega^{t}=\Omega \quad \text { and } \quad \operatorname{Im} \Omega>0 .
$$

For $n=1, \mathfrak{H}_{1}$ is the Poincaré upper half plane. The following two conditions are known to be equivalent for a lattice $\Lambda \subset W$ : i) The complex torus $W / \Lambda$ can be embedded, as a complex manifold, in a complex projective space $\mathbb{P}^{N}(\mathbb{C})$; ii) There exists a basis $\left(e_{1}, \ldots, e_{n}\right)$ of $W$, and a basis $\left(\lambda_{1}, \ldots, \lambda_{2 n}\right)$ of $\Lambda$ such that the matrix of $\left(\lambda_{1}, \ldots, \lambda_{2 n}\right)$ in the basis $\left(e_{1}, \ldots, e_{n}\right)$ is of the form

$$
\left(\Delta_{k}, \Omega\right),
$$

where $k=\left(k_{1}, \ldots, k_{n}\right)$ is a sequence of integers $k_{i} \in \mathbb{Z}, \Delta_{k}=\operatorname{diag}\left(k_{1}, \ldots, k_{n}\right)$ is a diagonal matrix, and $\Omega \in \mathfrak{H}_{n}$. A noncommutative torus $\mathbb{T}_{\theta}^{n}$ attached to a pair $(W, \Lambda)$ satisfying the equivalent conditions i) or ii) can be regarded as a noncommutative abelian variety. 


\section{Laplacian and its heat kernel}

In this section we consider the curved geometry of noncommutative 4-tori and analyse the corresponding Laplacian. We also recall the pseudodifferential calculus of [5] for these spaces and review the derivation of small time heat kernel expansions using this calculus.

3.1. Perturbed Laplacian on $\mathbb{T}_{\theta}^{4}$. We consider the construction of Section 2 for the noncommutative 4-torus $\mathbb{T}_{\theta}^{4}$, the matrix $\Omega=i I_{2 \times 2}$ in the Siegel upper half space $\mathfrak{H}_{2}$, and $k_{1}=k_{2}=1$. That is, we consider the complex structure on $\mathbb{T}_{\theta}^{4}$ which is introduced by setting $\partial, \bar{\partial}: C^{\infty}\left(\mathbb{T}_{\theta}^{4}\right) \rightarrow C^{\infty}\left(\mathbb{T}_{\theta}^{4}\right) \oplus C^{\infty}\left(\mathbb{T}_{\theta}^{4}\right)$ as

$$
\partial=\partial_{1} \oplus \partial_{2}, \quad \bar{\partial}=\bar{\partial}_{1} \oplus \bar{\partial}_{2},
$$

where

$$
\begin{array}{ll}
\partial_{1}=\delta_{1}-i \delta_{3}, & \partial_{2}=\delta_{2}-i \delta_{4}, \\
\bar{\partial}_{1}=\delta_{1}+i \delta_{3}, & \bar{\partial}_{2}=\delta_{2}+i \delta_{4} .
\end{array}
$$

We consider the inner product

$$
(a, b)=\varphi_{0}\left(b^{*} a\right), \quad a, b \in C\left(\mathbb{T}_{\theta}^{4}\right),
$$

and denote the Hilbert space completion of $C\left(\mathbb{T}_{\theta}^{4}\right)$ with respect to this inner product by $\mathcal{H}_{0}$. Since the derivations $\delta_{i}$ are formally selfadjoint with respect to the above inner product, we have

$$
\partial^{*} \partial=\delta_{1}^{2}+\delta_{2}^{2}+\delta_{3}^{2}+\delta_{4}^{2},
$$

which is the flat Laplacian $\triangle$ introduced in Section 2. The reason for this coincidence is that the underlying metric is Kähler when we have the non-perturbed standard volume form $\varphi_{0}$. Therefore, the ordinary Laplacian and the Dolbeault Laplacian agree with each other in this case.

In order to perturb the above Laplacian conformally, following [4, 12], we consider a selfadjoint element $h \in C^{\infty}\left(\mathbb{T}_{\theta}^{4}\right)$ and perturb the volume form $\varphi_{0}$ by replacing it with the linear functional $\varphi: C\left(\mathbb{T}_{\theta}^{4}\right) \rightarrow \mathbb{C}$ defined by

$$
\varphi(a)=\varphi_{0}\left(a e^{-2 h}\right), \quad a \in C\left(\mathbb{T}_{\theta}^{4}\right) .
$$

This is a non-tracial state which is a twisted trace, and satisfies the KMS condition at $\beta=1$ for the 1-parameter group $\left\{\sigma_{t}\right\}_{t \in \mathbb{R}}$ of inner automorphisms

$$
\sigma_{t}(a)=e^{2 i t h} a e^{-2 i t h}, \quad a \in C\left(\mathbb{T}_{\theta}^{4}\right) .
$$

The inner product associated with this linear functional is given by

$$
(a, b)_{\varphi}=\varphi\left(b^{*} a\right), \quad a, b \in C\left(\mathbb{T}_{\theta}^{4}\right) .
$$


We denote the Hilbert space completion of $C\left(\mathbb{T}_{\theta}^{4}\right)$ with respect to this inner product by $\mathcal{H}_{\varphi}$, and write the analogue of the de Rham differential on $\mathbb{T}_{\theta}^{4}$ as

$$
d=\partial \oplus \bar{\partial}: \mathcal{H}_{\varphi} \rightarrow \mathcal{H}_{\varphi}^{(1,0)} \oplus \mathcal{H}_{\varphi}^{(0,1)}
$$

Here, we view $d$ as an operator from $\mathcal{H}_{\varphi}$ to the corresponding Hilbert space completion of the analogue of 1-forms, namely the direct sum of the linear span of $\left\{a \partial b ; a, b \in C^{\infty}\left(\mathbb{T}_{\theta}^{4}\right)\right\}$ and $\left\{a \bar{\partial} b ; a, b \in C^{\infty}\left(\mathbb{T}_{\theta}^{4}\right)\right\}$.

Now we define the perturbed Laplacian

$$
\triangle_{\varphi}=d^{*} d,
$$

which is an unbounded operator acting in $\mathcal{H}_{\varphi}$. We will see in the following lemma that $\triangle_{\varphi}$ is anti-unitarily equivalent to a differential operator acting in $\mathcal{H}_{0}$, and because of this equivalence, we identify these operators with each other in the sequel.

Lemma 3.1. The perturbed Laplacian $\triangle_{\varphi}$ is anti-unitarily equivalent to the operator

$$
e^{h} \bar{\partial}_{1} e^{-h} \partial_{1} e^{h}+e^{h} \partial_{1} e^{-h} \bar{\partial}_{1} e^{h}+e^{h} \bar{\partial}_{2} e^{-h} \partial_{2} e^{h}+e^{h} \partial_{2} e^{-h} \bar{\partial}_{2} e^{h},
$$

acting in $\mathcal{H}_{0}$.

Proof. It follows easily from the argument given in the proof of the following lemma.

Let $h^{\prime}, h^{\prime \prime} \in C^{\infty}\left(\mathbb{T}_{\theta}^{4}\right)$ be selfadjoint elements, and $H, H_{0}, H_{1}$ respectively be the Hilbert space completion of $C\left(\mathbb{T}_{\theta}^{4}\right)$ with respect to the inner products defined by

$$
(a, b)=\varphi_{0}\left(b^{*} a\right), \quad(a, b)_{0}=\varphi_{0}\left(b^{*} a e^{-h^{\prime}}\right), \quad(a, b)_{1}=\varphi_{0}\left(b^{*} a e^{-h^{\prime \prime}}\right),
$$

for any $a, b \in C\left(\mathbb{T}_{\theta}^{4}\right)$. We recall the operator $\partial_{1}=\delta_{1}-i \delta_{3}: H \rightarrow H$ and its adjoint $\bar{\partial}_{1}=\delta_{1}+i \delta_{3}$.

Lemma 3.2. Let $\partial_{0,1}$ be the same map as $\partial_{1}$ viewed as an operator from $H_{0}$ to $H_{1}$. Then its adjoint is given by

$$
\partial_{0,1}^{*}(y)=\bar{\partial}_{1}\left(y e^{-h^{\prime \prime}}\right) e^{h^{\prime}},
$$

and the operator $\partial_{0,1}^{*} \partial_{0,1}: H_{0} \rightarrow H_{0}$ is anti-unitarily equivalent to

$$
e^{h^{\prime} / 2} \partial_{1} e^{-h^{\prime \prime}} \bar{\partial}_{1} e^{h^{\prime} / 2}: H \rightarrow H,
$$

where $e^{h^{\prime}}, e^{-h^{\prime \prime}}, e^{h^{\prime} / 2}$ act by left multiplication. 
Proof. Right multiplication by $e^{h^{\prime} / 2}$ extends to a unitary map from $H$ to $H_{0}$, which we denote by $W_{0}$. Similarly, right multiplication by $e^{-h^{\prime \prime} / 2}$ extends to a unitary map from $H_{1}$ to $H$, which will be denoted by $W_{1}$. So we have

$$
W_{1} \partial_{0,1} W_{0}=R_{e^{-h^{\prime \prime} / 2} \partial_{1}} R_{e^{h^{\prime} / 2}} .
$$

Therefore, we have

$$
W_{0}^{*} \partial_{0,1}^{*} W_{1}^{*} W_{1} \partial_{0,1} W_{0}=R_{e^{h^{\prime} / 2}} \bar{\partial}_{1} R_{e^{-h^{\prime \prime} / 2}} R_{e^{-h^{\prime \prime} / 2} \partial_{1}} R_{e^{h^{\prime} / 2}} .
$$

Thus $\partial_{0,1}^{*} \partial_{0,1}$ is unitarily equivalent to

$$
R_{e^{h^{\prime} / 2}} \bar{\partial}_{1} R_{e^{-h^{\prime \prime} / 2}} R_{e^{-h^{\prime \prime} / 2} \partial_{1}} R_{e^{h^{\prime} / 2}}
$$

Conjugating the latter with the anti-unitary involution $J(a)=a^{*}$, one can see that it is anti-unitarily equivalent to

$$
e^{h^{\prime} / 2} \partial_{1} e^{-h^{\prime \prime}} \bar{\partial}_{1} e^{h^{\prime} / 2}
$$

3.2. A pseudodifferential calculus for $\mathbb{T}_{\theta}^{4}$. A pseudodifferential calculus was developed in [5] for $C^{*}$-dynamical systems. Here we briefly recall this calculus for the canonical dynamical system defining the noncommutative 4-torus, and will use it in the sequel to apply heat kernel techniques $[19,12]$ to the perturbed Laplacian $\triangle_{\varphi}$ on $\mathbb{T}_{\theta}^{4}$.

A differential operator of order $m \in \mathbb{Z}_{\geq 0}$ on $\mathbb{T}_{\theta}^{4}$ is an operator of the form

$$
\sum_{|\ell| \leq m} a_{\ell} \delta_{1}^{\ell_{1}} \delta_{2}^{\ell_{2}} \delta_{3}^{\ell_{3}} \delta_{4}^{\ell_{4}}
$$

where $\ell=\left(\ell_{1}, \ell_{2}, \ell_{3}, \ell_{4}\right) \in \mathbb{Z}_{\geq 0}^{4},|\ell|=\ell_{1}+\ell_{2}+\ell_{3}+\ell_{4}, a_{\ell} \in C^{\infty}\left(\mathbb{T}_{\theta}^{4}\right)$. We first recall the definition of the operator valued symbols, using which, the notion of differential operators on $\mathbb{T}_{\theta}^{4}$ extends to the notion of pseudodifferential operators [5]. For convenience, we will use the notation $\partial_{j}$ for the partial derivatives $\frac{\partial}{\partial \xi_{j}}$ with respect to the coordinates $\xi=\left(\xi_{1}, \ldots, \xi_{4}\right) \in \mathbb{R}^{4}$, and for any $\ell \in \mathbb{Z}_{\geq 0}^{4}$, we denote $\ell_{1} ! \ell_{2} ! \ell_{3} ! \ell_{4}$ ! by $\ell !, \xi_{1}^{\ell_{1}} \xi_{2}^{\ell_{2}} \xi_{3}^{\ell_{3}} \xi_{4}^{\ell_{4}}$ by $\xi^{\ell}, \partial_{1}^{\ell_{1}} \partial_{2}^{\ell_{2}} \partial_{3}^{\ell_{3}} \partial_{4}^{\ell_{4}}$ by $\partial^{\ell}, \delta_{1}^{\ell_{1}} \delta_{2}^{\ell_{2}} \delta_{3}^{\ell_{3}} \delta_{4}^{\ell_{4}}$ by $\delta^{\ell}$, and $U_{1}^{\ell_{1}} U_{2}^{\ell_{2}} U_{3}^{\ell_{3}} U_{4}^{\ell_{4}}$ by $U^{\ell}$.

Definition 3.3. A smooth map $\rho: \mathbb{R}^{4} \rightarrow C^{\infty}\left(\mathbb{T}_{\theta}^{4}\right)$ is said to be a symbol of order $m \in \mathbb{Z}$, if for any set of non-negative integers $i, j \in \mathbb{Z}_{\geq 0}^{4}$, there exists a constant $c$ such that

$$
\left\|\partial^{j} \delta^{i}(\rho(\xi))\right\| \leq c(1+|\xi|)^{m-|j|},
$$

and if there exists a smooth map $k: \mathbb{R}^{4} \backslash\{0\} \rightarrow C^{\infty}\left(\mathbb{T}_{\theta}^{4}\right)$ such that

$$
\lim _{\lambda \rightarrow \infty} \lambda^{-m} \rho(\lambda \xi)=k(\xi), \quad \xi \in \mathbb{R}^{4} \backslash\{0\} .
$$


The space of symbols of order $m$ is denoted by $S_{m}$. The pseudodifferential operator $P_{\rho}: C^{\infty}\left(\mathbb{T}_{\theta}^{4}\right) \rightarrow C^{\infty}\left(\mathbb{T}_{\theta}^{4}\right)$ associated to a symbol $\rho \in S_{m}$ is given by

$$
P_{\rho}(a)=(2 \pi)^{-4} \iint e^{-i s \cdot \xi} \rho(\xi) \alpha_{s}(a) d s d \xi, \quad a \in C^{\infty}\left(\mathbb{T}_{\theta}^{4}\right),
$$

where $\left\{\alpha_{s}\right\}_{s \in \mathbb{R}^{4}}$ is the group of $C^{*}$-algebra automorphisms defined by

$$
\alpha_{s}\left(U^{\ell}\right)=e^{i s \cdot \ell} U^{\ell}, \quad \ell \in \mathbb{Z}^{4},
$$

which was explained in more generality in Subsection 2.1. For example, the differential operator $\sum a_{\ell} \delta^{\ell}$ is associated with the symbol $\sum a_{\ell} \xi^{\ell}$ via the above formula.

The pseudodifferential operators on $\mathbb{T}_{\theta}^{4}$ form an algebra and there is an asymptotic expansion for the symbol of the composition of two such operators. There is also an asymptotic formula for the symbol of the formal adjoint of a pseudodifferential operator, where the adjoint is taken with respect to the inner product given by (3.1). We explain this in the following proposition in which the relation $\rho \sim \sum_{j=0}^{\infty} \rho_{j}$ for given symbols $\rho, \rho_{j}$ means that for any $k \in \mathbb{Z}$, there exists an $N \in \mathbb{Z}_{\geq 0}$ such that $\rho-\sum_{j=0}^{n} \rho_{j} \in S_{k}$ for any $n>N$.

Proposition 3.4. Let $\rho \in S_{m}$ and $\rho^{\prime} \in S_{m^{\prime}}$. There exists a unique $\lambda \in S_{m+m^{\prime}}$ such that

$$
P_{\lambda}=P_{\rho} P_{\rho^{\prime}}
$$

Moreover,

$$
\lambda \sim \sum_{\ell \in \mathbb{Z}_{\geq 0}^{4}} \frac{1}{\ell !} \partial^{\ell} \rho(\xi) \delta^{\ell}\left(\rho^{\prime}(\xi)\right) .
$$

There is also a unique $\tau \in S_{m}$ such that $P_{\tau}$ is the formal adjoint of $P_{\rho}$, and

$$
\tau \sim \sum_{\ell \in \mathbb{Z}_{\geq 0}^{4}} \frac{1}{\ell !} \partial^{\ell} \delta^{\ell}(\rho(\xi))^{*} .
$$

Elliptic pseudodifferential operators on $\mathbb{T}_{\theta}^{4}$ are defined to be those whose symbols have the following property:

Definition 3.5. A symbol $\rho \in S_{m}$ is said to be elliptic if $\rho(\xi)$ is invertible for any $\xi \neq 0$, and if there exists a constant $c$ such that

$$
\left\|\rho(\xi)^{-1}\right\| \leq c(1+|\xi|)^{-m}
$$

when $|\xi|$ is sufficiently large.

As an example, the flat Laplacian $\Delta=\delta_{1}^{2}+\delta_{2}^{2}+\delta_{3}^{2}+\delta_{4}^{2}$ is an elliptic operator of order 2 since its symbol is $\rho(\xi)=\xi_{1}^{2}+\xi_{2}^{2}+\xi_{3}^{2}+\xi_{4}^{2}$, which satisfies the above criterion. 
3.3. Small time asymptotic expansion for $\operatorname{Trace}\left(e^{-t \Delta_{\varphi}}\right)$. Geometric invariants of a Riemannian manifold, such as its volume and scalar curvature, can be computed by considering small time heat kernel expansions of its Laplacian. Pseudodifferential calculus may be employed to compute the terms of such expansions (cf. [19]). One can use the pseudodifferential calculus of [5] to apply similar heat kernel techniques in order to compute geometric invariants of noncommutative spaces $[12,11,15,16,17]$. Here, we briefly explain this method and derive the small time asymptotic expansion for the trace of $e^{-t \Delta_{\varphi}}$, where $\triangle_{\varphi}$ is the perturbed Laplacian on $\mathbb{T}_{\theta}^{4}$ introduced in Subsection 3.1. First we need to compute the symbol of this differential operator.

Lemma 3.6. The symbol of $\triangle_{\varphi}$ is equal to $a_{2}(\xi)+a_{1}(\xi)+a_{0}(\xi)$, where

$$
\begin{aligned}
& a_{2}(\xi)=e^{h} \sum_{i=1}^{4} \xi_{i}^{2}, \quad a_{1}(\xi)=\sum_{i=1}^{4} \delta_{i}\left(e^{h}\right) \xi_{i}, \\
& a_{0}(\xi)=\sum_{i=1}^{4}\left(\delta_{i}^{2}\left(e^{h}\right)-\delta_{i}\left(e^{h}\right) e^{-h} \delta_{i}\left(e^{h}\right)\right) .
\end{aligned}
$$

Proof. It follows easily from the symbol calculus explained in Proposition 3.4.

Using the Cauchy integral formula, one has

$$
e^{-t \Delta_{\varphi}}=\frac{1}{2 \pi i} \int_{C} e^{-t \lambda}\left(\triangle_{\varphi}-\lambda\right)^{-1} d \lambda,
$$

where $C$ is a curve in the complex plane that goes around the non-negative real axis in such a way that

$$
e^{-t s}=\frac{1}{2 \pi i} \int_{C} e^{-t \lambda}(s-\lambda)^{-1} d \lambda, \quad s \geq 0 .
$$

Appealing to this formula, one can use the pseudodifferential calculus to employ similar arguments to those in [19] and derive an asymptotic expansion of the form

$$
\operatorname{Trace}\left(e^{-t \Delta_{\varphi}}\right) \sim t^{-2} \sum_{n=0}^{\infty} B_{2 n}\left(\triangle_{\varphi}\right) t^{n} \quad(t \rightarrow 0)
$$

That is, one can approximate $\left(\triangle_{\varphi}-\lambda\right)^{-1}$ by pseudodifferential operators $B_{\lambda}$ whose symbols are of the form

$$
b_{0}(\xi, \lambda)+b_{1}(\xi, \lambda)+b_{2}(\xi, \lambda)+\cdots,
$$

where for $j=0,1,2, \ldots, b_{j}(\xi, \lambda)$ is a symbol of order $-2-j$. 
Therefore, we have to use the calculus of symbols explained in Subsection 3.2 to solve the equation

$$
\left(b_{0}+b_{1}+b_{2}+\cdots\right)\left(\left(a_{2}-\lambda\right)+a_{1}+a_{0}\right) \sim 1 .
$$

Here, $\lambda$ is treated as a symbol of order 2 and we let $a_{2}^{\prime}=a_{2}-\lambda, a_{1}^{\prime}=a_{1}, a_{0}^{\prime}=a_{0}$. Then the above equation yields

$$
\sum_{\substack{j \in \mathbb{Z}_{\geq 0}, \ell \in \mathbb{Z}_{\geq 0}^{4} \\ k=0,1,2}} \frac{1}{\ell !} \partial^{\ell} b_{j} \delta^{\ell}\left(a_{k}^{\prime}\right) \sim 1 .
$$

Comparing symbols of the same order on both sides, one concludes that

$$
b_{0}=a_{2}^{\prime-1}=\left(a_{2}-\lambda\right)^{-1}=\left(e^{h} \sum_{i=1}^{4} \xi_{i}^{2}-\lambda\right)^{-1},
$$

and

$$
b_{n}=-\sum \frac{1}{\ell !} \partial^{\ell} b_{j} \delta^{\ell}\left(a_{k}\right) b_{0}, \quad n>0,
$$

where the summation is over all $0 \leq j<n, 0 \leq k \leq 2, \ell \in \mathbb{Z}_{\geq 0}^{4}$ such that $2+j+|\ell|-k=n$. Similar to $[19,12]$ one can use these symbols to approximate $e^{-t \Delta_{\varphi}}$ with suitable infinitely smoothing operators and derive the desired asymptotic expansion. We record this result in the following proposition.

Proposition 3.7. There is a small time asymptotic expansion

$$
\operatorname{Trace}\left(e^{-t \Delta_{\varphi}}\right) \sim t^{-2} \sum_{n=0}^{\infty} B_{2 n}\left(\triangle_{\varphi}\right) t^{n} \quad(t \rightarrow 0),
$$

where for each $n=0,1,2, \ldots$,

$$
B_{2 n}\left(\triangle_{\varphi}\right)=\frac{1}{2 \pi i} \iint_{C} e^{-\lambda} \varphi_{0}\left(b_{2 n}(\xi, \lambda)\right) d \lambda d \xi
$$

\section{Weyl's law and Connes' trace theorem}

A celebrated theorem of Weyl states that one can hear the volume of a closed Riemannian manifold $(M, g)$ from the asymptotic distribution of the eigenvalues of its Laplacian $\triangle_{g}$ acting on smooth functions on $M$. That is, if $0 \leq \lambda_{0} \leq \lambda_{1} \leq \lambda_{2} \leq \cdots$ are the eigenvalues of $\triangle_{g}$ counted with multiplicity and $N(\lambda)=\#\left\{\lambda_{j} \leq \lambda\right\}$ is the eigenvalue counting function then

$$
N(\lambda) \sim \frac{\operatorname{Vol}(M)}{(4 \pi)^{n / 2} \Gamma\left(\frac{n}{2}+1\right)} \lambda^{n / 2} \quad(\lambda \rightarrow \infty),
$$


where $n=\operatorname{dim} M$ and $\operatorname{Vol}(M)$ is the volume of $M$. An equivalent formulation of this result is the following asymptotic estimate for the eigenvalues:

$$
\lambda_{j} \sim \frac{4 \pi \Gamma\left(\frac{n}{2}+1\right)^{2 / n}}{\operatorname{Vol}(M)^{2 / n}} j^{2 / n} \quad(j \rightarrow \infty) .
$$

This readily shows that $\left(1+\triangle_{g}\right)^{-n / 2}$ is in the domain of the Dixmier trace and

$$
\operatorname{Tr}_{\omega}\left(\left(1+\triangle_{g}\right)^{-n / 2}\right)=\frac{\operatorname{Vol}(M)}{4 \pi^{n / 2} \Gamma\left(\frac{n}{2}+1\right)} .
$$

A generalization of this result is the trace theorem of [7] which states that the Dixmier trace and Wodzicki's noncommutative residue [22] coincide on pseudodifferential operators of order $-n$ acting on the sections of a vector bundle over $M$. In the sequel we will provide more explanations about the Dixmier trace, the noncommutative residue, and the trace theorem.

In this section, we establish the analogue of Weyl's law for $\mathbb{T}_{\theta}^{4}$ by studying the asymptotic distribution of the eigenvalues of the Laplacian $\triangle_{\varphi}$. We will then prove a trace theorem for $\mathbb{T}_{\theta}^{4}$. This is done by introducing a noncommutative residue on the algebra of classical pseudodifferential operators on $\mathbb{T}_{\theta}^{4}$ and showing that it coincides with the Dixmier trace on the pseudodifferential operators of order -4 . We closely follow the constructions and arguments given in $[17,18]$ for the noncommutative 2-torus, and because of similarities in the arguments, we provide essentials of the proofs rather briefly.

4.1. Asymptotic distribution of the eigenvalues of $\triangle_{\varphi}$. Let $0 \leq \lambda_{0} \leq \lambda_{1} \leq$ $\lambda_{2} \leq \cdots$ be the eigenvalues of $\Delta_{\varphi}$, counted with multiplicity. It follows from the asymptotic expansion

$$
\operatorname{Trace}\left(e^{-t \Delta_{\varphi}}\right)=\sum_{j=0}^{\infty} e^{-t \lambda_{j}} \sim t^{-2} \sum_{n=0}^{\infty} B_{2 n}\left(\triangle_{\varphi}\right) t^{n} \quad(t \rightarrow 0),
$$

derived in Proposition 3.7, that

$$
\lim _{t \rightarrow 0^{+}} t^{2} \sum e^{-t \lambda_{j}}=B_{0}\left(\triangle_{\varphi}\right) .
$$

It readily follows from Karamata's Tauberian theorem [1] that the corresponding eigenvalue counting function $N$ has the following asymptotic behavior:

$$
N(\lambda) \sim \frac{B_{0}\left(\triangle_{\varphi}\right)}{\Gamma(3)} \lambda^{2} \quad(\lambda \rightarrow \infty) .
$$

We establish the analogue of Weyl's law for $\mathbb{T}_{\theta}^{4}$ in the following theorem by computing $B_{0}\left(\triangle_{\varphi}\right)$ and observing that, up to a universal constant, it is equal to $\varphi(1)=\varphi_{0}\left(e^{-2 h}\right)$. 
Theorem 4.1. The eigenvalue counting function $N$ of the Laplacian $\triangle_{\varphi}$ on $\mathbb{T}_{\theta}^{4}$ satisfies

$$
N(\lambda) \sim \frac{\pi^{2} \varphi_{0}\left(e^{-2 h}\right)}{2} \lambda^{2} \quad(\lambda \rightarrow \infty) .
$$

Proof. Using Proposition 3.7, we have

$$
\begin{aligned}
B_{0}\left(\triangle_{\varphi}\right) & =\frac{1}{2 \pi i} \iint_{C} e^{-\lambda} \varphi_{0}\left(b_{0}(\xi, \lambda)\right) d \lambda d \xi \\
& =\varphi_{0}\left(\frac{1}{2 \pi i} \iint_{C} e^{-\lambda}\left(e^{h} \sum_{i=1}^{4} \xi_{i}^{2}-\lambda\right)^{-1} d \lambda d \xi\right) \\
& =\varphi_{0}\left(\int e^{-e^{h}\left(\xi_{1}^{2}+\cdots+\xi_{4}^{2}\right)} d \xi\right) \\
& =\pi^{2} \varphi_{0}\left(e^{-2 h}\right) .
\end{aligned}
$$

Thus, it follows from the above discussion that

$$
N(\lambda) \sim \frac{\pi^{2} \varphi_{0}\left(e^{-2 h}\right)}{\Gamma(3)} \lambda^{2}=\frac{\pi^{2} \varphi_{0}\left(e^{-2 h}\right)}{2} \lambda^{2} \quad(\lambda \rightarrow \infty) .
$$

A corollary of this theorem is that $\left(1+\triangle_{\varphi}\right)^{-2}$ is in the domain of the Dixmier trace. Before stating the corollary we quickly review the Dixmier trace and the noncommutative integral, following [8].

We denote the ideal of compact operators on a Hilbert space $\mathcal{H}$ by $\mathcal{K}(\mathcal{H})$. For any $T \in \mathcal{K}(\mathcal{H})$, let $\mu_{n}(T), n=1,2, \ldots$, denote the sequence of eigenvalues of its absolute value $|T|=\left(T^{*} T\right)^{\frac{1}{2}}$ written in decreasing order with multiplicity:

$$
\mu_{1}(T) \geq \mu_{2}(T) \geq \cdots \geq 0 .
$$

The Dixmier trace is a trace functional on an ideal of compact operators $\mathcal{L}^{1, \infty}(\mathcal{H})$ defined as

$$
\mathcal{L}^{1, \infty}(\mathcal{H})=\left\{T \in \mathcal{K}(\mathcal{H}) ; \quad \sum_{n=1}^{N} \mu_{n}(T)=O(\log N)\right\} .
$$

This ideal of operators is equipped with a natural norm:

$$
\|T\|_{1, \infty}:=\sup _{N \geq 2} \frac{1}{\log N} \sum_{n=1}^{N} \mu_{n}(T), \quad T \in \mathcal{L}^{1, \infty}(\mathcal{H}) .
$$

It is clear that trace class operators are automatically in $\mathcal{L}^{1, \infty}(\mathcal{H})$. The Dixmier trace of an operator $T \in \mathcal{L}^{1, \infty}(\mathcal{H})$ measures the logarithmic divergence of its ordinary 
trace. More precisely, for any positive operator $T \in \mathcal{L}^{1, \infty}(\mathcal{H})$ we are interested in the limiting behavior of the sequence

$$
\frac{1}{\log N} \sum_{n=1}^{N} \mu_{n}(T), \quad N=2,3, \ldots .
$$

While by our assumption this sequence is bounded, its usual limit may not exist and must be replaced by a suitable generalized limit. The limiting procedure is carried out by means of a state on a $C^{*}$-algebra. Recall that a state on a $C^{*}$-algebra is a non-zero positive linear functional on the algebra.

To define the Dixmier trace of a positive operator $T \in \mathcal{L}^{1, \infty}(\mathcal{H})$, consider the partial trace

$$
\operatorname{Trace}_{N}(T)=\sum_{n=1}^{N} \mu_{n}(T), \quad N=1,2, \ldots,
$$

and its piecewise affine interpolation denoted by $\operatorname{Trace}_{r}(T)$ for $r \in[1, \infty)$. Then let

$$
\tau_{\Lambda}(T):=\frac{1}{\log \Lambda} \int_{e}^{\Lambda} \frac{\operatorname{Trace}_{r}(T)}{\log r} \frac{d r}{r}, \quad \Lambda \in[e, \infty),
$$

be the Cesàro mean of the function $\operatorname{Trace}_{r}(T) / \log r$ over the multiplicative group $\mathbb{R}_{>0}$. Now choosing a normalized state $\omega: C_{b}[e, \infty) \rightarrow \mathbb{C}$ on the algebra of bounded continuous functions on $[e, \infty)$ such that $\omega(f)=0$ for all $f$ vanishing at $\infty$, the Dixmier trace of $T \geq 0$ is defined as

$$
\operatorname{Tr}_{\omega}(T)=\omega\left(\tau_{\Lambda}(T)\right)
$$

Then one can extend $\operatorname{Tr}_{\omega}$ to all of $\mathcal{L}^{1, \infty}(\mathcal{H})$ by linearity.

The resulting linear functional $\operatorname{Tr}_{\omega}$ is a positive trace on $\mathcal{L}^{(1, \infty)}(\mathcal{H})$ which in general depends on the limiting procedure $\omega$. The operators $T \in \mathcal{L}^{1, \infty}(\mathcal{H})$ whose Dixmier trace $\operatorname{Tr}_{\omega}(T)$ is independent of the choice of the state $\omega$ are called measurable and we will denote their Dixmier trace by $f T$. If for a compact positive operator $T$ we have

$$
\mu_{n}(T) \sim \frac{c}{n} \quad(n \rightarrow \infty),
$$

where $c$ is a constant, then $T$ is measurable and $f T=c$. We use this fact in the proof of the following corollary of Theorem 4.1.

Corollary 4.2. The operator $\left(1+\triangle_{\varphi}\right)^{-2}$, where $\triangle_{\varphi}$ is the perturbed Laplacian on $\mathbb{T}_{\theta}^{4}$, is a measurable operator in $\mathcal{L}^{1, \infty}\left(\mathcal{H}_{0}\right)$, and

$$
f\left(1+\triangle_{\varphi}\right)^{-2}=\frac{\pi^{2}}{2} \varphi_{0}\left(e^{-2 h}\right)
$$


Proof. It follows from the asymptotic behavior (4.1) of the eigenvalue counting function $N$ that the eigenvalues $\lambda_{j}$ of $\triangle_{\varphi}$ satisfy

$$
\lambda_{j} \sim \frac{\sqrt{2}}{\pi \varphi_{0}\left(e^{-2 h}\right)^{1 / 2}} j^{1 / 2} \quad(j \rightarrow \infty) .
$$

Therefore, using the above fact, it easily follows that $\left(1+\triangle_{\varphi}\right)^{-2}$ is measurable and $f\left(1+\triangle_{\varphi}\right)^{-2}=\frac{\pi^{2}}{2} \varphi_{0}\left(e^{-2 h}\right)$.

4.2. A noncommutative residue for $\mathbb{T}_{\theta}^{4}$. Let $M$ be a closed smooth manifold of dimension $n$. Wodzicki defined a trace functional on the algebra of pseudodifferential operators of arbitrary order on $M$, and proved that it was the only non-trivial trace [22]. This functional, denoted by Res, is called the noncommutative residue.

The noncommutative residue of a classical pseudodifferential operator $P$ acting on smooth sections of a vector bundle $E$ over $M$ is defined as

$$
\operatorname{Res}(P)=(2 \pi)^{-n} \int_{S^{*} M} \operatorname{tr}\left(\rho_{-n}(x, \xi)\right) d x d \xi,
$$

where $S^{*} M \subset T^{*} M$ is the unit cosphere bundle on $M$ and $\rho_{-n}$ is the component of order $-n$ of the complete symbol of $P$.

Similar to [18], we define a noncommutative residue on the algebra of classical pseudodifferential operators on $\mathbb{T}_{\theta}^{4}$, which is a natural analogue of the Wodzicki residue.

Definition 4.3. A pseudodifferential symbol $\rho \in S_{m}$ on $\mathbb{T}_{\theta}^{4}$ is said to be classical if there is an asymptotic expansion of the form

$$
\rho(\xi) \sim \sum_{j=0}^{\infty} \rho_{m-j}(\xi) \quad(\xi \rightarrow \infty),
$$

where each $\rho_{m-j}: \mathbb{R}^{4} \backslash\{0\} \rightarrow C^{\infty}\left(\mathbb{T}_{\theta}^{4}\right)$ is smooth and positively homogeneous of order $m-j$. Given such a symbol, we define the noncommutative residue of the corresponding pseudodifferential operator $P_{\rho}$ as

$$
\operatorname{res}\left(P_{\rho}\right)=\int_{\mathbb{S}^{3}} \varphi_{0}\left(\rho_{-4}(\xi)\right) d \Omega,
$$

where $d \Omega$ is the standard invariant measure on the unit sphere in $\mathbb{R}^{4}$.

We note that, as shown in [18], the homogeneous terms in the expansion of any classical pseudodifferential symbol are uniquely determined. Thus, there is no ambiguity in the above definition.

In the following theorem we identify all continuous trace functionals on the algebra of classical pseudodifferential operators on $\mathbb{T}_{\theta}^{4}$. A linear functional on this 
algebra is said to be continuous if it vanishes on the operators whose symbols are of sufficiently small order. First we state two lemmas which will be used in the proof of the following theorem. One can prove these lemmas by similar arguments to those given in [18].

Lemma 4.4. Let $f: \mathbb{R}^{4} \backslash\{0\} \rightarrow C^{\infty}\left(\mathbb{T}_{\theta}^{4}\right)$ be a smooth map which is positively homogeneous of order $m \in \mathbb{Z}$. If $m \neq-4$, or if $m=-4$ and $\int_{\mathbb{S}^{3}} f d \Omega=0$, then one can write $f=\sum_{i=1}^{4} \partial_{i}\left(h_{i}\right)$, for some smooth maps $h_{i}: \mathbb{R}^{4} \backslash\{0\} \rightarrow C^{\infty}\left(\mathbb{T}_{\theta}^{4}\right)$.

Lemma 4.5. Let $\sigma_{j} \in S_{m_{j}}, j=0,1,2, \ldots$, be a sequence of pseudodifferential symbols on $\mathbb{T}_{\theta}^{4}$ such that $\lim m_{j}=-\infty$. There exists a symbol $\sigma$ such that $\sigma \sim$ $\sum_{j=0}^{\infty} \sigma_{j}$.

Theorem 4.6. The noncommutative residue res is a trace, and up to multiplication by a constant, it is the unique continuous trace on the algebra of classical pseudodifferential operators on $\mathbb{T}_{\theta}^{4}$.

Proof. Let $\rho, \rho^{\prime}: \mathbb{R}^{4} \rightarrow C^{\infty}\left(\mathbb{T}_{\theta}^{4}\right)$ be classical symbols with asymptotic expansions

$$
\rho(\xi) \sim \sum_{j=0}^{\infty} \rho_{n-j}(\xi), \quad \rho^{\prime}(\xi) \sim \sum_{k=0}^{\infty} \rho_{n^{\prime}-k}(\xi) \quad(\xi \rightarrow \infty),
$$

where $\rho_{n-j}$ is homogeneous of order $n-j$ and $\rho_{n^{\prime}-k}$ is homogeneous of order $n^{\prime}-k$. Using the calculus of symbols explained in Proposition 3.4 and the trace property of $\varphi_{0}$, we have:

$$
\begin{aligned}
\operatorname{res}\left(P_{\rho} P_{\rho^{\prime}}-\right. & \left.P_{\rho^{\prime}} P_{\rho}\right) \\
& =\int_{\mathbb{S}^{3}} \varphi_{0}\left(\sum \frac{1}{\ell !}\left(\partial^{\ell}\left(\rho_{n-j}\right) \delta^{\ell}\left(\rho_{n^{\prime}-k}^{\prime}\right)-\delta^{\ell}\left(\rho_{n-j}\right) \partial^{\ell}\left(\rho_{n^{\prime}-k}^{\prime}\right)\right)\right) d \Omega,
\end{aligned}
$$

where the summation is over all $j, k \in \mathbb{Z}_{\geq 0}$ and $\ell \in \mathbb{Z}_{\geq 0}^{4}$ such that $n+n^{\prime}-j-k-$ $|\ell|=-4$. One can write each $\partial^{\ell}\left(\rho_{n-j}\right) \delta^{\ell}\left(\rho_{n^{\prime}-k}^{\prime}\right)-\delta^{\ell}\left(\rho_{n-j}\right) \partial^{\ell}\left(\rho_{n^{\prime}-k}^{\prime}\right)$ in the above integral as

$$
\sum_{i=1}^{4}\left(\partial_{i}\left(f_{i}\right)+\delta_{i}\left(g_{i}\right)\right),
$$

for some $f_{i}, g_{i}: \mathbb{R}^{4} \backslash\{0\} \rightarrow C^{\infty}\left(\mathbb{T}_{\theta}^{4}\right)$, where each $f_{i}$ is positively homogeneous of order -3 . Thus, using Lemma 5.1.3 on page 208 of [21] and the fact that $\varphi_{0} \circ \delta_{i}=0$, we have

$$
\operatorname{res}\left(P_{\rho} P_{\rho^{\prime}}-P_{\rho^{\prime}} P_{\rho}\right)=0 .
$$

This proves the trace property of res.

In order to prove the uniqueness, assume that $\psi$ is a continuous trace on the algebra of classical symbols on $\mathbb{T}_{\theta}^{4}$. For any classical symbol $\rho$, the symbol of $P_{\xi_{i}} P_{\rho}-P_{\rho} P_{\xi_{i}}$ is equivalent to $\delta_{i}(\rho)$. Since $\psi$ is a trace, it follows that

$$
\psi\left(P_{\delta_{i}(\rho)}\right)=0 .
$$


Since we can write

$$
\rho=\varphi_{0}(\rho)+\sum_{i=1}^{4} \delta_{i}\left(\rho_{i}\right),
$$

for some symbols $\rho_{i}$, equation (4.2) implies that

$$
\psi\left(P_{\rho}\right)=\psi\left(P_{\varphi_{0}(\rho)}\right) .
$$

Also, by considering the symbol of $P_{\rho} P_{U_{i}}-P_{U_{i}} P_{\rho}$ and using similar arguments to those in [18], one can conclude that

$$
\psi\left(P_{\partial_{i}(\rho)}\right)=0 .
$$

Now we consider the asymptotic expansion

$$
\rho(\xi) \sim \sum_{j=0}^{\infty} \rho_{n-j}(\xi), \quad(\xi \rightarrow \infty),
$$

where $\rho_{n-j}$ is positively homogeneous of order $n-j$. By using Lemmas 4.4 and 4.5 , and setting $r=\operatorname{Vol}\left(\mathbb{S}^{3}\right)^{-1} \int_{\mathbb{S}^{3}} \rho_{-4} d \Omega$, we can write

$$
\begin{aligned}
\rho & \sim \rho_{-4}+\sum_{n-j \neq-4} \rho_{n-j} \\
& =\frac{r}{|\xi|^{4}}+\left(\rho_{-4}-\frac{r}{|\xi|^{4}}\right)+\sum_{n-j \neq-4} \sum_{i=1}^{4} \partial_{i}\left(\rho_{n-j, i}\right) \\
& \sim \frac{r}{|\xi|^{4}}+\sum_{i=1}^{4} \partial_{i}\left(\rho_{-4, i}\right)+\sum_{i=1}^{4} \partial_{i}\left(\sum_{n-j \neq-4} \rho_{n-j, i}\right),
\end{aligned}
$$

for some smooth maps $\rho_{-4, i}, \rho_{n-j, i}: \mathbb{R}^{4} \backslash\{0\} \rightarrow C^{\infty}\left(\mathbb{T}_{\theta}^{4}\right)$. Now we can use (4.3), (4.4), (4.5) to conclude that

$$
\psi\left(P_{\rho}\right)=\psi\left(P_{r /\left|\xi^{4}\right|}\right)=\psi\left(P_{\varphi_{0}\left(r /\left|\xi^{4}\right|\right)}\right)=\varphi_{0}(r) \psi\left(P_{1 /\left|\xi^{4}\right|}\right)=\frac{\psi\left(P_{1 /\left|\xi^{4}\right|}\right)}{\operatorname{Vol}\left(\mathbb{S}^{3}\right)} \operatorname{res}\left(P_{\rho}\right) .
$$

4.3. A trace theorem for $\mathbb{T}_{\theta}^{4}$. As above, let $M$ be a closed smooth manifold of dimension $n$. The restriction of the Wodzicki residue Res to pseudodifferential operators of order $-n$ was discovered independently by Guillemin and its properties were studied in [20]. In general, unlike the Dixmier trace, Res is not a positive linear functional. However, its restriction to pseudodifferential operators of order $-n$ is positive. One of the main results proved in [7] is that if $E$ is a smooth vector bundle 
on $M$ then the Dixmier trace $\operatorname{Tr}_{\omega}$ and Res coincide on pseudodifferential operators of order $-n$ acting on $L^{2}$ sections of $E$. In fact it is proved that such operators $P$ are measurable operators in $\mathcal{L}^{1, \infty}\left(L^{2}(M, E)\right)$ and

$$
f P=\frac{1}{n} \operatorname{Res}(P) \text {. }
$$

In the following theorem we establish the analogue of this result for the noncommutative 4-torus $\mathbb{T}_{\theta}^{4}$.

Theorem 4.7. Let $\rho$ be a classical pseudodifferential symbol of order -4 on $\mathbb{T}_{\theta}^{4}$. Then $P_{\rho}$ is a measurable operator in $\mathcal{L}^{1, \infty}\left(\mathcal{H}_{0}\right)$, and under the assumption that all nonzero entries of $\theta$ are irrational, we have

$$
f P_{\rho}=\frac{1}{4} \operatorname{res}\left(P_{\rho}\right)
$$

Proof. In order to show that $P_{\rho} \in \mathcal{L}^{1, \infty}\left(\mathcal{H}_{0}\right)$, we write $P_{\rho}=A(1+\triangle)^{-2}$, where $\triangle=\delta_{1}^{2}+\cdots+\delta_{4}^{2}$ is the flat Laplacian, and $A=P_{\rho}(1+\triangle)^{2}$ is a pseudodifferential operator of order 0 . Since $A$ is a bounded operator on $\mathcal{H}_{0}$ and it was shown in Corollary 4.2 that $(1+\triangle)^{-2} \in \mathcal{L}^{1, \infty}\left(\mathcal{H}_{0}\right)$, it follows that $P_{\rho}$ is in the domain of the Dixmier trace. Using a similar argument, one can see that any pseudodifferential operator of order -5 on $\mathbb{T}_{\theta}^{4}$ is in the kernel of the Dixmier trace. Therefore, if we write

$$
\rho(\xi) \sim \rho^{\prime}(\xi)+\sum_{j=1}^{\infty} \rho_{-4-j}(\xi) \quad(\xi \rightarrow \infty),
$$

where $\rho^{\prime}$ and $\rho_{-4-j}$ are respectively positively homogeneous of order -4 and $-4-j$, then

$$
\operatorname{Tr}_{\omega}\left(P_{\rho}\right)=\operatorname{Tr}_{\omega}\left(P_{\rho^{\prime}}\right)
$$

Also, since the symbol of $P_{\rho^{\prime}} P_{U_{i}}-P_{U_{i}} P_{\rho^{\prime}}$ is equivalent to the symbol of $P_{\rho^{\prime} U_{i}}-$ $P_{U_{i} \rho^{\prime}}$ modulo a symbol of order -5 , and $\operatorname{Tr}_{\omega}$ is a trace, for $i=1, \ldots 4$, we have

$$
\operatorname{Tr}_{\omega}\left(P_{\rho^{\prime} U_{i}}\right)=\operatorname{Tr}_{\omega}\left(P_{U_{i} \rho^{\prime}}\right) .
$$

It follows from this observation that if $f: \mathbb{R}^{4} \backslash\{0\} \rightarrow \mathbb{C}$ is smooth and positively homogeneous of order -4 , then $\operatorname{Tr}_{\omega}\left(P_{f U^{\ell}}\right)=0$ if $\ell \neq 0 \in \mathbb{Z}^{4}$. Therefore, similar to the argument given in [17] one can write the following expansion with rapidly decreasing coefficients

$$
\rho^{\prime}(\xi)=\sum_{\ell \in \mathbb{Z}^{4}} \rho_{\ell}^{\prime}(\xi) U^{\ell}
$$

and conclude that

$$
\operatorname{Tr}_{\omega}\left(P_{\rho^{\prime}}\right)=\operatorname{Tr}_{\omega}\left(P_{\rho_{0}^{\prime}}\right),
$$

where, as noted above, $\rho_{0}^{\prime}=\varphi_{0} \circ \rho^{\prime}: \mathbb{R}^{4} \backslash\{0\} \rightarrow \mathbb{C}$. 
Note that one has to use the fact that $\operatorname{Tr}_{\omega}$ is continuous with respect to the uniform norm of symbols of order -4 , namely that, if we let $q_{m}(\xi)=\rho^{\prime}(\xi)-$ $\sum_{|\ell| \leq m} a_{\ell}(\xi) U^{\ell}$, then

$$
\lim _{m \rightarrow \infty} \operatorname{Tr}_{\omega}\left(P_{q_{m}}\right)=0 .
$$

Since $\rho_{0}^{\prime}$ is a smooth complex-valued function on $\mathbb{R}^{4} \backslash\{0\}$ which is positively homogeneous of order -4 , in order to analyze $\operatorname{Tr}_{\omega}\left(P_{\rho_{0}^{\prime}}\right)$, we define a linear functional $\mu$ on the space of continuous complex-valued functions on $\mathbb{S}^{3}$, as follows. Given a smooth function $f: \mathbb{S}^{3} \rightarrow \mathbb{C}$, we denote its positively homogeneous extension of order -4 to $\mathbb{R}^{4} \backslash\{0\}$ by $\tilde{f}$, and define $\mu(f)=\operatorname{Tr}_{\omega}\left(P_{\tilde{f}}\right)$. Using the continuity property mentioned above, $\mu$ extends to the space of continuous functions on $\mathbb{S}^{3}$. Also using positivity of $\operatorname{Tr}_{\omega}$, one can see that $\mu$ is a positive linear functional. Thus, it follows from the Reisz representation theorem that $\mu$ is given by integration against a Borel measure on $\mathbb{S}^{3}$. This measure is rotation invariant, which can be shown by using the trace property of $\operatorname{Tr}_{\omega}$ and the fact that for any rotation $T$ of $\mathbb{R}^{4}$ and any pseudodifferential symbol $\sigma$ on $\mathbb{R}^{4}$ we have

$$
P_{\sigma(T x, T \xi)}=\mathcal{U}^{-1} P_{\sigma(x, \xi)} \mathcal{U}
$$

where $\mathcal{U}$ is the unitary operator $\mathcal{U}(g)=g \circ T^{-1}, g \in C_{c}^{\infty}\left(\mathbb{R}^{4}\right)$. Therefore $\mu$ is given by integration against a constant multiple of the standard invariant measure on $\mathbb{S}^{3}$. Denoting this constant by $c$, identities (4.6), (4.7) imply that

$$
\operatorname{Tr}_{\omega}\left(P_{\rho}\right)=\operatorname{Tr}_{\omega}\left(P_{\rho^{\prime}}\right)=\operatorname{Tr}_{\omega}\left(P_{\rho_{0}^{\prime}}\right)=\mu\left(\left.\rho_{0}^{\prime}\right|_{\mathbb{S}^{3}}\right)=\left.c \int_{\mathbb{S}^{3}} \rho_{0}^{\prime}\right|_{\mathbb{S}^{3}} d \Omega=c \operatorname{res}\left(P_{\rho}\right) .
$$

The constant $c$ can be fixed by considering the flat Laplacian $\triangle=\sum_{i=1}^{4} \delta_{i}^{2}$. According to corollary 4.2, we have $\operatorname{Tr}_{\omega}\left((1+\triangle)^{-2}\right)=\pi^{2} / 2$. On the other hand, considering the fact that the term of order -4 in the asymptotic expansion of the symbol of $(1+\triangle)^{-2}$ is $|\xi|^{-4}$, we have res $\left((1+\triangle)^{-2}\right)=2 \pi^{2}$. Therefore $c=1 / 4$.

\section{Scalar curvature and Einstein-Hilbert action}

Let $(M, g)$ be a smooth compact manifold of dimension $n \geq 2$, and $\triangle_{g}$ be the Laplacian acting on smooth functions on $M$. For any $t>0$, the operator $e^{-t \Delta g}$ is an infinitely smoothing operator, and there is an asymptotic expansion for its kernel $K(t, x, y)$, which is of the form

$$
K(t, x, y) \sim \frac{e^{-\operatorname{dist}(x, y)^{2} / 4 t}}{(4 \pi t)^{n / 2}}\left(u_{0}(x, y)+u_{1}(x, y) t+u_{2}(x, y) t^{2}+\cdots\right) \quad(t \rightarrow 0) .
$$

The coefficients $u_{i}$ are smooth functions defined in a neighborhood of the diagonal in $M \times M$. The kernel $K$ is called the heat kernel since it is the fundamental 
solution of the heat operator $\partial_{t}+\triangle_{g}$, and the $u_{i}$ are called the heat kernel coefficients. These coefficients are well-studied and are often known under the names of people who made the major contributions in the study, namely that, they are called both Minakshisundaram-Pleijel coefficients and Seeley-De Witt coefficients. Minakshisundaram and Pleijel derived the above asymptotic expansion by using the transport equation method of Hadamard. An approach, which is followed in the noncommutative case in $[12,11,15,16,17]$, is to use pseudodifferential calculus to derive such asymptotic expansions. For a clear account of this approach and a detailed discussion of the local geometric information that are encoded in heat coefficients, we refer the reader to [19] and the references therein.

A crucial point that is used to define and compute the scalar curvature for noncommutative spaces $[9,11,16]$ is that, up to a universal factor, the restriction of $u_{1}$ to the diagonal gives the scalar curvature of $M$. This, via the Mellin transform, allows to have a spectral definition for scalar curvature in terms of values or residues of spectral zeta functions. That is, if for instance the dimension of $M$ is 4, the scalar curvature is the unique $R \in C^{\infty}(M)$ (up to a universal constant) such that

$$
\operatorname{res}_{s=1} \operatorname{Trace}\left(f \triangle_{g}^{-s}\right)=\int_{M} f R d v o l_{g},
$$

for any $f \in C^{\infty}(M)$.

In this section we define the scalar curvature for $\mathbb{T}_{\theta}^{4}$ equipped with the perturbed Laplacian $\triangle_{\varphi}$, and compute the functions that give a local expression for the curvature. Then we consider the analogue of the Einstein-Hilbert action $\int_{M} R d v o l_{g}$. We find a local expression for this action as well, and show that its extremum is attained if and only if the Weyl factor is a constant, which is equivalent to having a metric with constant curvature.

5.1. Scalar curvature for $\mathbb{T}_{\theta}^{4}$. Following $[9,11,16]$ and the above discussion, we define the scalar curvature of $\mathbb{T}_{\theta}^{4}$ equipped with the perturbed Laplacian $\triangle_{\varphi}$ as follows. (See also [2] and [14] for variants, and [13] where a perturbative form of the Gauss-Bonnet theorem for terms up to second order in the perturbation parameter is verified.)

Definition 5.1. The scalar curvature of the noncommutative 4-torus equipped with the perturbed volume form is the unique element $R \in C^{\infty}\left(\mathbb{T}_{\theta}^{4}\right)$ such that

$$
\operatorname{res}_{s=1} \operatorname{Trace}\left(a \triangle_{\varphi}^{-s}\right)=\varphi_{0}(a R),
$$

for any $a \in C^{\infty}\left(\mathbb{T}_{\theta}^{4}\right)$.

We follow the method employed in $[12,11,16]$ to find a local expression for the scalar curvature $R$. For the sake of completeness, we explain this procedure in the following proposition. 
Proposition 5.2. The scalar curvature $R$ is equal to

$$
\frac{1}{2 \pi i} \int_{\mathbb{R}^{4}} \int_{C} e^{-\lambda} b_{2}(\xi, \lambda) d \lambda d \xi,
$$

where $b_{2}$ is the term of order -4 of the pseudodifferential symbol of the parametrix of $\triangle_{\varphi}-\lambda$, given in Subsection 3.3.

Proof. Using the Mellin transform we have

$$
a \triangle_{\varphi}^{-s}=\frac{1}{\Gamma(s)} \int_{0}^{\infty} a\left(e^{-t \triangle_{\varphi}}-P\right) t^{s-1} d t, \quad a \in C^{\infty}\left(\mathbb{T}_{\theta}^{4}\right), \quad \Re(s)>0,
$$

where $P$ denotes the orthogonal projection on $\operatorname{Ker}\left(\triangle_{\varphi}\right)$.

Appealing to the Cauchy integral formula (3.2) and using similar arguments to those explained in Subsection 3.3 (cf. [19, 12]), one can derive an asymptotic expansion of the form

$$
\operatorname{Trace}\left(a e^{-t \Delta_{\varphi}}\right) \sim t^{-2} \sum_{n=0}^{\infty} B_{2 n}\left(a, \triangle_{\varphi}\right) t^{n} \quad(t \rightarrow 0) .
$$

Using this asymptotic expansion one can see that the zeta function

$$
\zeta_{a}(s)=\operatorname{Trace}\left(a \triangle_{\varphi}^{-s}\right), \quad \Re(s) \gg 0,
$$

has a meromorphic extension to the whole plane with a simple pole at 1 , and

$$
\operatorname{res}_{s=1} \zeta_{a}(s)=B_{2}\left(a, \triangle_{\varphi}\right)
$$

On the other hand, there are explicit formulas for the coefficients of the above asymptotic expansion. In particular we have

$$
B_{2}\left(a, \triangle_{\varphi}\right)=\frac{1}{2 \pi i} \iint_{C} e^{-\lambda} \varphi_{0}\left(a b_{2}(\xi, \lambda)\right) d \lambda d \xi .
$$

We directly compute $b_{2}$ using (3.3) and in order to compute

$$
\frac{1}{2 \pi i} \int_{\mathbb{R}^{4}} \int_{C} e^{-\lambda} b_{2}(\xi, \lambda) d \lambda d \xi,
$$

we use a homogeneity argument for the contour integral (cf. $[12,11])$ and pass to the spherical coordinates

$$
\begin{array}{lll}
\xi_{1}=r \sin (\psi) \sin (\phi) \cos (\theta), & \xi_{2}=r \sin (\psi) \sin (\phi) \sin (\theta), \\
\xi_{3}=r \sin (\psi) \cos (\phi), & \xi_{4}=r \cos (\psi),
\end{array}
$$


with $0 \leq r<\infty, 0 \leq \psi<\pi, 0 \leq \phi<\pi, 0 \leq \theta<2 \pi$. After working out the integrations with respect to the angles $\psi, \phi, \theta$, we obtain the following terms up to an overall factor of $-\pi^{2}$ :

$$
\begin{aligned}
& +4 r^{9} e^{h} b_{0} b_{0} b_{0} \delta_{1}\left(e^{h}\right) e^{h} b_{0} b_{0} \delta_{1}\left(e^{h}\right) b_{0}+2 r^{9} e^{h} b_{0} b_{0} e^{h} b_{0} b_{0} \delta_{1}\left(e^{h}\right) b_{0} \delta_{1}\left(e^{h}\right) b_{0} \\
& +4 r^{9} e^{h} b_{0} b_{0} \delta_{1}\left(e^{h}\right) e^{h} b_{0} b_{0} b_{0} \delta_{1}\left(e^{h}\right) b_{0}+2 r^{9} e^{h} b_{0} b_{0} \delta_{1}\left(e^{h}\right) e^{h} b_{0} b_{0} \delta_{1}\left(e^{h}\right) b_{0} b_{0} \\
& +4 r^{9} e^{h} b_{0} e^{h} b_{0} b_{0} b_{0} \delta_{1}\left(e^{h}\right) b_{0} \delta_{1}\left(e^{h}\right) b_{0}+2 r^{9} e^{h} b_{0} e^{h} b_{0} b_{0} \delta_{1}\left(e^{h}\right) b_{0} b_{0} \delta_{1}\left(e^{h}\right) b_{0} \\
& +2 r^{9} e^{h} b_{0} e^{h} b_{0} b_{0} \delta_{1}\left(e^{h}\right) b_{0} \delta_{1}\left(e^{h}\right) b_{0} b_{0}+6 r^{9} e^{h} e^{h} b_{0} b_{0} b_{0} b_{0} \delta_{1}\left(e^{h}\right) b_{0} \delta_{1}\left(e^{h}\right) b_{0} \\
& +2 r^{9} e^{h} e^{h} b_{0} b_{0} b_{0} \delta_{1}\left(e^{h}\right) b_{0} b_{0} \delta_{1}\left(e^{h}\right) b_{0}+2 r^{9} e^{h} e^{h} b_{0} b_{0} b_{0} \delta_{1}\left(e^{h}\right) b_{0} \delta_{1}\left(e^{h}\right) b_{0} b_{0} \\
& -r^{7} b_{0} b_{0} \delta_{1}\left(e^{h}\right) e^{h} b_{0} b_{0} \delta_{1}\left(e^{h}\right) b_{0}-2 r^{7} b_{0} \delta_{1}\left(e^{h}\right) e^{h} b_{0} b_{0} b_{0} \delta_{1}\left(e^{h}\right) b_{0} \\
& -r^{7} b_{0} \delta_{1}\left(e^{h}\right) e^{h} b_{0} b_{0} \delta_{1}\left(e^{h}\right) b_{0} b_{0}-16 r^{7} e^{h} b_{0} b_{0} b_{0} \delta_{1}\left(e^{h}\right) b_{0} \delta_{1}\left(e^{h}\right) b_{0} \\
& -r^{7} e^{h} b_{0} b_{0} e^{h} b_{0} b_{0} \delta_{1} \delta_{1}\left(e^{h}\right) b_{0}-8 r^{7} e^{h} b_{0} b_{0} \delta_{1}\left(e^{h}\right) b_{0} b_{0} \delta_{1}\left(e^{h}\right) b_{0} \\
& -8 r^{7} e^{h} b_{0} b_{0} \delta_{1}\left(e^{h}\right) b_{0} \delta_{1}\left(e^{h}\right) b_{0} b_{0}-2 r^{7} e^{h} b_{0} e^{h} b_{0} b_{0} b_{0} \delta_{1} \delta_{1}\left(e^{h}\right) b_{0} \\
& -r^{7} e^{h} b_{0} e^{h} b_{0} b_{0} \delta_{1} \delta_{1}\left(e^{h}\right) b_{0} b_{0}-3 r^{7} e^{h} e^{h} b_{0} b_{0} b_{0} b_{0} \delta_{1} \delta_{1}\left(e^{h}\right) b_{0} \\
& -r^{7} e^{h} e^{h} b_{0} b_{0} b_{0} \delta_{1} \delta_{1}\left(e^{h}\right) b_{0} b_{0}+5 / 2 r^{5} b_{0} b_{0} \delta_{1}\left(e^{h}\right) b_{0} \delta_{1}\left(e^{h}\right) b_{0} \\
& +2 r^{3} b_{0} b_{0} \delta_{1}\left(e^{h}\right) e^{-h} \delta_{1}\left(e^{h}\right) b_{0}+5 / 2 r^{5} b_{0} \delta_{1}\left(e^{h}\right) b_{0} b_{0} \delta_{1}\left(e^{h}\right) b_{0} \\
& +5 / 2 r^{5} b_{0} \delta_{1}\left(e^{h}\right) b_{0} \delta_{1}\left(e^{h}\right) b_{0} b_{0}+2 r^{3} b_{0} \delta_{1}\left(e^{h}\right) e^{-h} \delta_{1}\left(e^{h}\right) b_{0} b_{0} \\
& +6 r^{5} e^{h} b_{0} b_{0} b_{0} \delta_{1} \delta_{1}\left(e^{h}\right) b_{0}+3 r^{5} e^{h} b_{0} b_{0} \delta_{1} \delta_{1}\left(e^{h}\right) b_{0} b_{0} \\
& -2 r^{3} b_{0} b_{0} \delta_{1} \delta_{1}\left(e^{h}\right) b_{0}-2 r^{3} b_{0} \delta_{1} \delta_{1}\left(e^{h}\right) b_{0} b_{0} \\
& +4 r^{9} e^{h} b_{0} b_{0} b_{0} \delta_{2}\left(e^{h}\right) e^{h} b_{0} b_{0} \delta_{2}\left(e^{h}\right) b_{0}+2 r^{9} e^{h} b_{0} b_{0} e^{h} b_{0} b_{0} \delta_{2}\left(e^{h}\right) b_{0} \delta_{2}\left(e^{h}\right) b_{0} \\
& +4 r^{9} e^{h} b_{0} b_{0} \delta_{2}\left(e^{h}\right) e^{h} b_{0} b_{0} b_{0} \delta_{2}\left(e^{h}\right) b_{0}+2 r^{9} e^{h} b_{0} b_{0} \delta_{2}\left(e^{h}\right) e^{h} b_{0} b_{0} \delta_{2}\left(e^{h}\right) b_{0} b_{0} \\
& +4 r^{9} e^{h} b_{0} e^{h} b_{0} b_{0} b_{0} \delta_{2}\left(e^{h}\right) b_{0} \delta_{2}\left(e^{h}\right) b_{0}+2 r^{9} e^{h} b_{0} e^{h} b_{0} b_{0} \delta_{2}\left(e^{h}\right) b_{0} b_{0} \delta_{2}\left(e^{h}\right) b_{0} \\
& +2 r^{9} e^{h} b_{0} e^{h} b_{0} b_{0} \delta_{2}\left(e^{h}\right) b_{0} \delta_{2}\left(e^{h}\right) b_{0} b_{0}+6 r^{9} e^{h} e^{h} b_{0} b_{0} b_{0} b_{0} \delta_{2}\left(e^{h}\right) b_{0} \delta_{2}\left(e^{h}\right) b_{0} \\
& +2 r^{9} e^{h} e^{h} b_{0} b_{0} b_{0} \delta_{2}\left(e^{h}\right) b_{0} b_{0} \delta_{2}\left(e^{h}\right) b_{0}+2 r^{9} e^{h} e^{h} b_{0} b_{0} b_{0} \delta_{2}\left(e^{h}\right) b_{0} \delta_{2}\left(e^{h}\right) b_{0} b_{0} \\
& -r^{7} b_{0} b_{0} \delta_{2}\left(e^{h}\right) e^{h} b_{0} b_{0} \delta_{2}\left(e^{h}\right) b_{0}-2 r^{7} b_{0} \delta_{2}\left(e^{h}\right) e^{h} b_{0} b_{0} b_{0} \delta_{2}\left(e^{h}\right) b_{0} \\
& -r^{7} b_{0} \delta_{2}\left(e^{h}\right) e^{h} b_{0} b_{0} \delta_{2}\left(e^{h}\right) b_{0} b_{0}-16 r^{7} e^{h} b_{0} b_{0} b_{0} \delta_{2}\left(e^{h}\right) b_{0} \delta_{2}\left(e^{h}\right) b_{0} \\
& -r^{7} e^{h} b_{0} b_{0} e^{h} b_{0} b_{0} \delta_{2} \delta_{2}\left(e^{h}\right) b_{0}-8 r^{7} e^{h} b_{0} b_{0} \delta_{2}\left(e^{h}\right) b_{0} b_{0} \delta_{2}\left(e^{h}\right) b_{0} \\
& -8 r^{7} e^{h} b_{0} b_{0} \delta_{2}\left(e^{h}\right) b_{0} \delta_{2}\left(e^{h}\right) b_{0} b_{0}-2 r^{7} e^{h} b_{0} e^{h} b_{0} b_{0} b_{0} \delta_{2} \delta_{2}\left(e^{h}\right) b_{0} \\
& -r^{7} e^{h} b_{0} e^{h} b_{0} b_{0} \delta_{2} \delta_{2}\left(e^{h}\right) b_{0} b_{0}-3 r^{7} e^{h} e^{h} b_{0} b_{0} b_{0} b_{0} \delta_{2} \delta_{2}\left(e^{h}\right) b_{0} \\
& -r^{7} e^{h} e^{h} b_{0} b_{0} b_{0} \delta_{2} \delta_{2}\left(e^{h}\right) b_{0} b_{0}+5 / 2 r^{5} b_{0} b_{0} \delta_{2}\left(e^{h}\right) b_{0} \delta_{2}\left(e^{h}\right) b_{0} \\
& +2 r^{3} b_{0} b_{0} \delta_{2}\left(e^{h}\right) e^{-h} \delta_{2}\left(e^{h}\right) b_{0}+5 / 2 r^{5} b_{0} \delta_{2}\left(e^{h}\right) b_{0} b_{0} \delta_{2}\left(e^{h}\right) b_{0} \\
& +5 / 2 r^{5} b_{0} \delta_{2}\left(e^{h}\right) b_{0} \delta_{2}\left(e^{h}\right) b_{0} b_{0}+2 r^{3} b_{0} \delta_{2}\left(e^{h}\right) e^{-h} \delta_{2}\left(e^{h}\right) b_{0} b_{0}
\end{aligned}
$$


$+6 r^{5} e^{h} b_{0} b_{0} b_{0} \delta_{2} \delta_{2}\left(e^{h}\right) b_{0}+3 r^{5} e^{h} b_{0} b_{0} \delta_{2} \delta_{2}\left(e^{h}\right) b_{0} b_{0}$

$-2 r^{3} b_{0} b_{0} \delta_{2} \delta_{2}\left(e^{h}\right) b_{0}-2 r^{3} b_{0} \delta_{2} \delta_{2}\left(e^{h}\right) b_{0} b_{0}$

$+4 r^{9} e^{h} b_{0} b_{0} b_{0} \delta_{3}\left(e^{h}\right) e^{h} b_{0} b_{0} \delta_{3}\left(e^{h}\right) b_{0}+2 r^{9} e^{h} b_{0} b_{0} e^{h} b_{0} b_{0} \delta_{3}\left(e^{h}\right) b_{0} \delta_{3}\left(e^{h}\right) b_{0}$

$+4 r^{9} e^{h} b_{0} b_{0} \delta_{3}\left(e^{h}\right) e^{h} b_{0} b_{0} b_{0} \delta_{3}\left(e^{h}\right) b_{0}+2 r^{9} e^{h} b_{0} b_{0} \delta_{3}\left(e^{h}\right) e^{h} b_{0} b_{0} \delta_{3}\left(e^{h}\right) b_{0} b_{0}$

$+4 r^{9} e^{h} b_{0} e^{h} b_{0} b_{0} b_{0} \delta_{3}\left(e^{h}\right) b_{0} \delta_{3}\left(e^{h}\right) b_{0}+2 r^{9} e^{h} b_{0} e^{h} b_{0} b_{0} \delta_{3}\left(e^{h}\right) b_{0} b_{0} \delta_{3}\left(e^{h}\right) b_{0}$

$+2 r^{9} e^{h} b_{0} e^{h} b_{0} b_{0} \delta_{3}\left(e^{h}\right) b_{0} \delta_{3}\left(e^{h}\right) b_{0} b_{0}+6 r^{9} e^{h} e^{h} b_{0} b_{0} b_{0} b_{0} \delta_{3}\left(e^{h}\right) b_{0} \delta_{3}\left(e^{h}\right) b_{0}$

$+2 r^{9} e^{h} e^{h} b_{0} b_{0} b_{0} \delta_{3}\left(e^{h}\right) b_{0} b_{0} \delta_{3}\left(e^{h}\right) b_{0}+2 r^{9} e^{h} e^{h} b_{0} b_{0} b_{0} \delta_{3}\left(e^{h}\right) b_{0} \delta_{3}\left(e^{h}\right) b_{0} b_{0}$

$-r^{7} b_{0} b_{0} \delta_{3}\left(e^{h}\right) e^{h} b_{0} b_{0} \delta_{3}\left(e^{h}\right) b_{0}-2 r^{7} b_{0} \delta_{3}\left(e^{h}\right) e^{h} b_{0} b_{0} b_{0} \delta_{3}\left(e^{h}\right) b_{0}$

$-r^{7} b_{0} \delta_{3}\left(e^{h}\right) e^{h} b_{0} b_{0} \delta_{3}\left(e^{h}\right) b_{0} b_{0}-16 r^{7} e^{h} b_{0} b_{0} b_{0} \delta_{3}\left(e^{h}\right) b_{0} \delta_{3}\left(e^{h}\right) b_{0}$

$-r^{7} e^{h} b_{0} b_{0} e^{h} b_{0} b_{0} \delta_{3} \delta_{3}\left(e^{h}\right) b_{0}-8 r^{7} e^{h} b_{0} b_{0} \delta_{3}\left(e^{h}\right) b_{0} b_{0} \delta_{3}\left(e^{h}\right) b_{0}$

$-8 r^{7} e^{h} b_{0} b_{0} \delta_{3}\left(e^{h}\right) b_{0} \delta_{3}\left(e^{h}\right) b_{0} b_{0}-2 r^{7} e^{h} b_{0} e^{h} b_{0} b_{0} b_{0} \delta_{3} \delta_{3}\left(e^{h}\right) b_{0}$

$-r^{7} e^{h} b_{0} e^{h} b_{0} b_{0} \delta_{3} \delta_{3}\left(e^{h}\right) b_{0} b_{0}-3 r^{7} e^{h} e^{h} b_{0} b_{0} b_{0} b_{0} \delta_{3} \delta_{3}\left(e^{h}\right) b_{0}$

$-r^{7} e^{h} e^{h} b_{0} b_{0} b_{0} \delta_{3} \delta_{3}\left(e^{h}\right) b_{0} b_{0}+5 / 2 r^{5} b_{0} b_{0} \delta_{3}\left(e^{h}\right) b_{0} \delta_{3}\left(e^{h}\right) b_{0}$

$+2 r^{3} b_{0} b_{0} \delta_{3}\left(e^{h}\right) e^{-h} \delta_{3}\left(e^{h}\right) b_{0}+5 / 2 r^{5} b_{0} \delta_{3}\left(e^{h}\right) b_{0} b_{0} \delta_{3}\left(e^{h}\right) b_{0}$

$+5 / 2 r^{5} b_{0} \delta_{3}\left(e^{h}\right) b_{0} \delta_{3}\left(e^{h}\right) b_{0} b_{0}+2 r^{3} b_{0} \delta_{3}\left(e^{h}\right) e^{-h} \delta_{3}\left(e^{h}\right) b_{0} b_{0}$

$+6 r^{5} e^{h} b_{0} b_{0} b_{0} \delta_{3} \delta_{3}\left(e^{h}\right) b_{0}+3 r^{5} e^{h} b_{0} b_{0} \delta_{3} \delta_{3}\left(e^{h}\right) b_{0} b_{0}$

$-2 r^{3} b_{0} b_{0} \delta_{3} \delta_{3}\left(e^{h}\right) b_{0}-2 r^{3} b_{0} \delta_{3} \delta_{3}\left(e^{h}\right) b_{0} b_{0}$

$+4 r^{9} e^{h} b_{0} b_{0} b_{0} \delta_{4}\left(e^{h}\right) e^{h} b_{0} b_{0} \delta_{4}\left(e^{h}\right) b_{0}+2 r^{9} e^{h} b_{0} b_{0} e^{h} b_{0} b_{0} \delta_{4}\left(e^{h}\right) b_{0} \delta_{4}\left(e^{h}\right) b_{0}$

$+4 r^{9} e^{h} b_{0} b_{0} \delta_{4}\left(e^{h}\right) e^{h} b_{0} b_{0} b_{0} \delta_{4}\left(e^{h}\right) b_{0}+2 r^{9} e^{h} b_{0} b_{0} \delta_{4}\left(e^{h}\right) e^{h} b_{0} b_{0} \delta_{4}\left(e^{h}\right) b_{0} b_{0}$

$+4 r^{9} e^{h} b_{0} e^{h} b_{0} b_{0} b_{0} \delta_{4}\left(e^{h}\right) b_{0} \delta_{4}\left(e^{h}\right) b_{0}+2 r^{9} e^{h} b_{0} e^{h} b_{0} b_{0} \delta_{4}\left(e^{h}\right) b_{0} b_{0} \delta_{4}\left(e^{h}\right) b_{0}$

$+2 r^{9} e^{h} b_{0} e^{h} b_{0} b_{0} \delta_{4}\left(e^{h}\right) b_{0} \delta_{4}\left(e^{h}\right) b_{0} b_{0}+6 r^{9} e^{h} e^{h} b_{0} b_{0} b_{0} b_{0} \delta_{4}\left(e^{h}\right) b_{0} \delta_{4}\left(e^{h}\right) b_{0}$

$+2 r^{9} e^{h} e^{h} b_{0} b_{0} b_{0} \delta_{4}\left(e^{h}\right) b_{0} b_{0} \delta_{4}\left(e^{h}\right) b_{0}+2 r^{9} e^{h} e^{h} b_{0} b_{0} b_{0} \delta_{4}\left(e^{h}\right) b_{0} \delta_{4}\left(e^{h}\right) b_{0} b_{0}$

$-r^{7} b_{0} b_{0} \delta_{4}\left(e^{h}\right) e^{h} b_{0} b_{0} \delta_{4}\left(e^{h}\right) b_{0}-2 r^{7} b_{0} \delta_{4}\left(e^{h}\right) e^{h} b_{0} b_{0} b_{0} \delta_{4}\left(e^{h}\right) b_{0}$

$-r^{7} b_{0} \delta_{4}\left(e^{h}\right) e^{h} b_{0} b_{0} \delta_{4}\left(e^{h}\right) b_{0} b_{0}-16 r^{7} e^{h} b_{0} b_{0} b_{0} \delta_{4}\left(e^{h}\right) b_{0} \delta_{4}\left(e^{h}\right) b_{0}$

$-r^{7} e^{h} b_{0} b_{0} e^{h} b_{0} b_{0} \delta_{4} \delta_{4}\left(e^{h}\right) b_{0}-8 r^{7} e^{h} b_{0} b_{0} \delta_{4}\left(e^{h}\right) b_{0} b_{0} \delta_{4}\left(e^{h}\right) b_{0}$

$-8 r^{7} e^{h} b_{0} b_{0} \delta_{4}\left(e^{h}\right) b_{0} \delta_{4}\left(e^{h}\right) b_{0} b_{0}-2 r^{7} e^{h} b_{0} e^{h} b_{0} b_{0} b_{0} \delta_{4} \delta_{4}\left(e^{h}\right) b_{0}$

$-r^{7} e^{h} b_{0} e^{h} b_{0} b_{0} \delta_{4} \delta_{4}\left(e^{h}\right) b_{0} b_{0}-3 r^{7} e^{h} e^{h} b_{0} b_{0} b_{0} b_{0} \delta_{4} \delta_{4}\left(e^{h}\right) b_{0}$

$-r^{7} e^{h} e^{h} b_{0} b_{0} b_{0} \delta_{4} \delta_{4}\left(e^{h}\right) b_{0} b_{0}+5 / 2 r^{5} b_{0} b_{0} \delta_{4}\left(e^{h}\right) b_{0} \delta_{4}\left(e^{h}\right) b_{0}$

$+2 r^{3} b_{0} b_{0} \delta_{4}\left(e^{h}\right) e^{-h} \delta_{4}\left(e^{h}\right) b_{0}+5 / 2 r^{5} b_{0} \delta_{4}\left(e^{h}\right) b_{0} b_{0} \delta_{4}\left(e^{h}\right) b_{0}$

$+5 / 2 r^{5} b_{0} \delta_{4}\left(e^{h}\right) b_{0} \delta_{4}\left(e^{h}\right) b_{0} b_{0}+2 r^{3} b_{0} \delta_{4}\left(e^{h}\right) e^{-h} \delta_{4}\left(e^{h}\right) b_{0} b_{0}$ 


$$
\begin{aligned}
& +6 r^{5} e^{h} b_{0} b_{0} b_{0} \delta_{4} \delta_{4}\left(e^{h}\right) b_{0}+3 r^{5} e^{h} b_{0} b_{0} \delta_{4} \delta_{4}\left(e^{h}\right) b_{0} b_{0} \\
& -2 r^{3} b_{0} b_{0} \delta_{4} \delta_{4}\left(e^{h}\right) b_{0}-2 r^{3} b_{0} \delta_{4} \delta_{4}\left(e^{h}\right) b_{0} b_{0} .
\end{aligned}
$$

We use the rearrangement lemma of [11] to integrate the above terms with respect to $r$ from 0 to $\infty$. We recall this lemma here:

Lemma 5.3. For any $m=\left(m_{0}, m_{1}, \ldots, m_{\ell}\right) \in \mathbb{Z}_{>0}^{\ell+1}$ and elements $\rho_{1}, \ldots, \rho_{\ell} \in$ $C^{\infty}\left(\mathbb{T}_{\theta}^{4}\right)$, we have

$$
\int_{0}^{\infty} \frac{u^{|m|-2}}{\left(e^{h} u+1\right)^{m_{0}}} \prod_{1}^{\ell} \rho_{j}\left(e^{h} u+1\right)^{-m_{j}} d u=e^{-(|m|-1) h} F_{m}\left(\Delta_{(1)}, \ldots, \Delta_{(\ell)}\right)\left(\prod_{1}^{\ell} \rho_{j}\right),
$$

where

$$
\begin{gathered}
F_{m}\left(u_{1}, \ldots, u_{\ell}\right)=\int_{0}^{\infty} \frac{x^{|m|-2}}{(x+1)^{m_{0}}} \prod_{1}^{\ell}\left(x \prod_{1}^{j} u_{h}+1\right)^{-m_{j}} d x, \\
\Delta(a)=e^{-h} a e^{h}, \quad a \in C\left(\mathbb{T}_{\theta}^{4}\right) .
\end{gathered}
$$

Here, $\Delta_{(j)}$ signifies the action of $\Delta$ on $\rho_{j}$.

Then, we use the identities (cf. $[11,16]$ )

$$
\begin{aligned}
e^{-h} \delta_{i}\left(e^{h}\right) & =g_{1}(\Delta)\left(\delta_{i}(h)\right), \\
e^{-h} \delta_{i}^{2}\left(e^{h}\right) & =g_{1}(\Delta)\left(\delta_{i}^{2}(h)\right)+2 g_{2}\left(\Delta_{(1)}, \Delta_{(2)}\right)\left(\delta_{i}(h) \delta_{i}(h)\right),
\end{aligned}
$$

where

$$
g_{1}(u)=\frac{u-1}{\log u}, \quad g_{2}(u, v)=\frac{u(v-1) \log (u)-(u-1) \log (v)}{\log (u) \log (v)(\log (u)+\log (v))},
$$

and obtain the final formula for the scalar curvature in terms of $\nabla=\log \Delta$ and $h$, which is recorded in the following theorem.

Theorem 5.4. The scalar curvature $R$ of $\mathbb{T}_{\theta}^{4}$, up to a factor of $\pi^{2}$, is equal to

$$
e^{-h} K(\nabla)\left(\sum_{i=1}^{4} \delta_{i}^{2}(h)\right)+e^{-h} H\left(\nabla_{(1)}, \nabla_{(2)}\right)\left(\sum_{i=1}^{4} \delta_{i}(h)^{2}\right)
$$

where

$$
\begin{aligned}
K(s) & =\frac{1-e^{-s}}{2 s} \\
H(s, t) & =-\frac{e^{-s-t}\left(\left(-e^{s}-3\right) s\left(e^{t}-1\right)+\left(e^{s}-1\right)\left(3 e^{t}+1\right) t\right)}{4 s t(s+t)} .
\end{aligned}
$$


We analyse the functions $K, H$, which describe the scalar curvature of $\mathbb{T}_{\theta}^{4}$, as follows. The Taylor expansion of $K$ at 0 is of the form

$$
K(s)=\frac{1}{2}-\frac{s}{4}+\frac{s^{2}}{12}-\frac{s^{3}}{48}+\frac{s^{4}}{240}-\frac{s^{5}}{1440}+O\left(s^{6}\right) .
$$

We have $\lim _{s \rightarrow-\infty} K(s)=\infty, \lim _{s \rightarrow \infty} K(s)=0$, and here is the graph of this function:

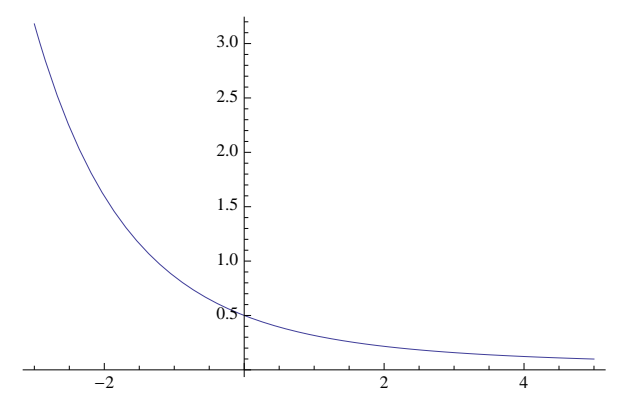

Graph of the function $K$.

The function $H$ has the following Taylor expansion at $(0,0)$ :

$$
\begin{gathered}
H(s, t)=\left(-\frac{1}{4}+\frac{t}{24}-\frac{t^{3}}{480}+O\left(t^{4}\right)\right)+s\left(\frac{5}{24}-\frac{t}{16}+\frac{t^{2}}{80}-\frac{t^{3}}{576}+O\left(t^{4}\right)\right) \\
+s^{2}\left(-\frac{1}{12}+\frac{7 t}{240}-\frac{t^{2}}{144}+\frac{5 t^{3}}{4032}+O\left(t^{4}\right)\right) \\
+s^{3}\left(\frac{11}{480}-\frac{5 t}{576}+\frac{t^{2}}{448}-\frac{t^{3}}{2304}+O\left(t^{4}\right)\right)+O\left(s^{4}\right) .
\end{gathered}
$$

Here is the graph of $H$ in a neighborhood of the origin:

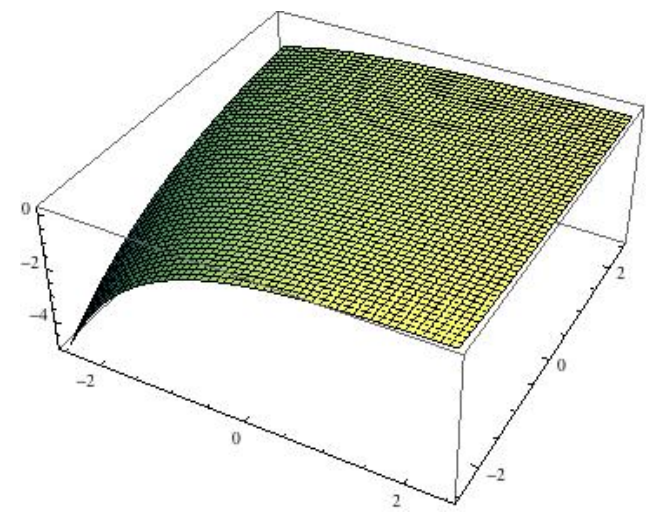

Graph of the function $H$. 
This function is not bounded from below on the main diagonal as for

$$
H(s, s)=-\frac{e^{-2 s}\left(e^{s}-1\right)^{2}}{4 s^{2}},
$$

we have $\lim _{s \rightarrow-\infty} H(s, s)=-\infty$, and $\lim _{s \rightarrow \infty} H(s, s)=0$. At 0 we have the Taylor expansion

$$
H(s, s)=-\frac{1}{4}+\frac{s}{4}-\frac{7 s^{2}}{48}+\frac{s^{3}}{16}-\frac{31 s^{4}}{1440}+\frac{s^{5}}{160}+O\left(s^{6}\right),
$$

and here is the graph of this function:

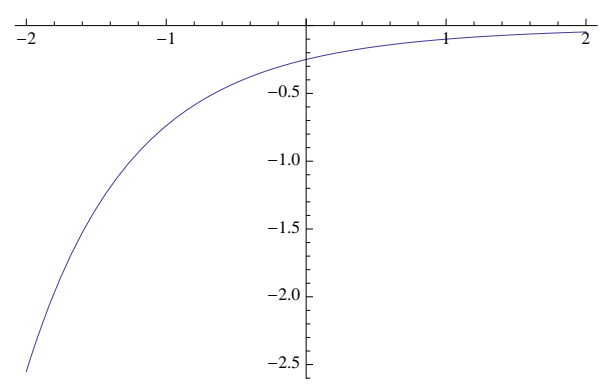

Graph of the map $s \mapsto H(s, s)$.

On the other diagonal, $H$ is neither bounded below nor bounded above as we have

$$
H(s,-s)=\frac{-4 s-3 e^{-s}+e^{s}+2}{4 s^{2}},
$$

which implies that $\lim _{s \rightarrow-\infty} H(s,-s)=-\infty, \lim _{s \rightarrow \infty} H(s,-s)=\infty$. This function has the following Taylor expansion at 0

$$
H(s,-s)=-\frac{1}{4}+\frac{s}{6}-\frac{s^{2}}{48}+\frac{s^{3}}{120}-\frac{s^{4}}{1440}+\frac{s^{5}}{5040}+O\left(s^{6}\right),
$$

and here is its graph:

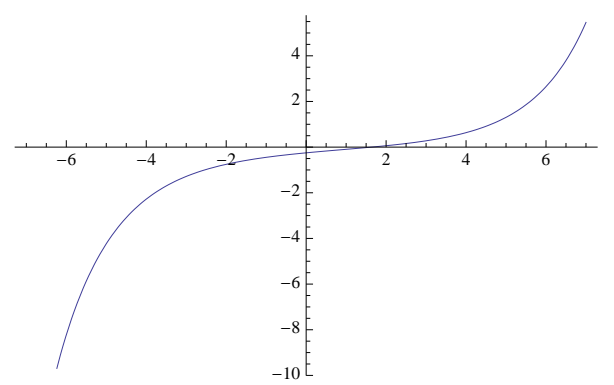

Graph of the map $s \mapsto H(s,-s)$. 
Remark 5.5. Since $K(0)=1 / 2$ and $H(0,0)=-1 / 4$, in the commutative case, the scalar curvature given by $(5.1)$ reduces to

$$
\frac{\pi^{2}}{2} \sum_{i=1}^{4}\left(\delta_{i}^{2}(h)-\frac{1}{2} \delta_{i}(h)^{2}\right)
$$

which, up to a normalization factor, is the scalar curvature of the ordinary 4-torus equipped with the metric $e^{-h}\left(d x_{1}^{2}+\cdots+d x_{4}^{2}\right)$.

5.2. Einstein-Hilbert action for $\mathbb{T}_{\theta}^{4}$. A natural analogue of the Einstein-Hilbert action for $\mathbb{T}_{\theta}^{4}$ is $\varphi_{0}(R)$, where $R$ is the scalar curvature given by (5.1). In the following theorem we find an explicit formula for this action.

Theorem 5.6. A local expression for the Einstein-Hilbert action for $\mathbb{T}_{\theta}^{4}$, up to a factor of $\pi^{2}$, is given by

$$
\varphi_{0}(R)=\frac{1}{2} \varphi_{0}\left(\sum_{i=1}^{4} e^{-h} \delta_{i}^{2}(h)\right)+\varphi_{0}\left(\sum_{i=1}^{4} G(\nabla)\left(e^{-h} \delta_{i}(h)\right) \delta_{i}(h)\right),
$$

where

$$
G(s)=\frac{-4 s-3 e^{-s}+e^{s}+2}{4 s^{2}} .
$$

Proof. Let us recall from Theorem 5.4 that up to a factor of $\pi^{2}$

$$
R=e^{-h} K(\nabla)\left(\sum_{i=1}^{4} \delta_{i}^{2}(h)\right)+e^{-h} H\left(\nabla_{(1)}, \nabla_{(2)}\right)\left(\sum_{i=1}^{4} \delta_{i}(h)^{2}\right) .
$$

Writing $K$ as a Fourier transform

$$
K(s)=\int e^{-i u s} f(u) d u,
$$

we have

$$
\varphi_{0}\left(e^{-h} K(\nabla)\left(\delta_{i}^{2}(h)\right)\right)=\int \varphi_{0}\left(e^{-h} \Delta^{-i u}\left(\delta_{i}^{2}(h)\right)\right) f(u) d u=K(0) \varphi_{0}\left(e^{-h} \delta_{i}^{2}(h)\right) .
$$

Also, by writing $H$ as a Fourier transform

$$
H(s, t)=\int e^{-i(s u+t v)} g(u, v) d u d v,
$$


we have

$$
\begin{aligned}
\varphi_{0}\left(e^{-h} H\left(\nabla_{(1)}, \nabla_{(2)}\right)\left(\delta_{i}(h)^{2}\right)\right) & =\int \varphi_{0}\left(e^{-h} \Delta^{-i u}\left(\delta_{i}(h)\right) \Delta^{-i v}\left(\delta_{i}(h)\right)\right) g(u, v) d u d v \\
& =\int \varphi_{0}\left(\Delta^{-i(u-v)}\left(e^{-h} \delta_{i}(h)\right) \delta_{i}(h)\right) g(u, v) d u d v \\
& =\varphi_{0}\left(H(\nabla,-\nabla)\left(e^{-h} \delta_{i}(h)\right) \delta_{i}(h)\right) .
\end{aligned}
$$

Therefore

$$
\varphi_{0}(R)=\frac{1}{2} \varphi_{0}\left(\sum_{i=1}^{4} e^{-h} \delta_{i}^{2}(h)\right)+\varphi_{0}\left(\sum_{i=1}^{4} G(\nabla)\left(e^{-h} \delta_{i}(h)\right) \delta_{i}(h)\right),
$$

where

$$
G(s)=H(s,-s)=\frac{-4 s-3 e^{-s}+e^{s}+2}{4 s^{2}} .
$$

5.3. Extremum of the Einstein-Hilbert action for $\mathbb{T}_{\theta}^{4}$. We show that the Einstein-Hilbert action $\varphi_{0}(R)$ attains its maximum if and only if the Weyl factor $e^{-h}$ is a constant. This is done by combining the two terms in the explicit formula (5.2) for $\varphi_{0}(R)$, and observing that it can be expressed by a non-negative function. We note that the function $G$ in (5.2), which was analysed in Subsection 5.1, is neither bounded below nor bounded above.

Theorem 5.7. The maximum of the Einstein-Hilbert action is equal to 0 , and it is attained if and only if the Weyl factor is a constant. That is, for any Weyl factor $e^{-h}, h=h^{*} \in C^{\infty}\left(\mathbb{T}_{\theta}^{4}\right)$, we have

$$
\varphi_{0}(R) \leq 0,
$$

and the equality happens if and only if $h$ is a constant.

Proof. We can combine the two terms in (5.2) as follows. We have

$$
\begin{aligned}
\varphi_{0}\left(e^{-h} \delta_{i}^{2}(h)\right) & =-\varphi_{0}\left(\delta_{i}\left(e^{-h}\right) \delta_{i}(h)\right) \\
& =\varphi_{0}\left(e^{-h} \delta_{i}\left(e^{h}\right) e^{-h} \delta_{i}(h)\right) \\
& =\varphi_{0}\left(e^{-h} \delta_{i}(h) e^{-h} \delta_{i}\left(e^{h}\right)\right) \\
& =\varphi_{0}\left(e^{-h} \delta_{i}(h) \frac{\Delta-1}{\log \Delta}\left(\delta_{i}(h)\right)\right) \\
& =\varphi_{0}\left(e^{-h} \delta_{i}(h) \frac{e^{\nabla}-1}{\nabla}\left(\delta_{i}(h)\right)\right) \\
& =\varphi_{0}\left(e^{-h} \frac{e^{-\nabla}-1}{-\nabla}\left(\delta_{i}(h)\right) \delta_{i}(h)\right) .
\end{aligned}
$$


The last equality follows from the fact that for any entire function $\mathrm{F}$, one has

$$
\varphi_{0}\left(e^{-h} a F(\nabla)(b)\right)=\varphi_{0}\left(e^{-h} F(-\nabla)(a) b\right), \quad a, b \in C\left(\mathbb{T}_{\theta}^{4}\right) .
$$

Therefore we can write (5.2) as

$$
\begin{aligned}
\varphi_{0}(R) & =\sum_{i=1}^{4} \varphi_{0}\left(\frac{1}{2} e^{-h} \frac{e^{-\nabla}-1}{-\nabla}\left(\delta_{i}(h)\right) \delta_{i}(h)+G(\nabla)\left(e^{-h} \delta_{i}(h)\right) \delta_{i}(h)\right) \\
& =\sum_{i=1}^{4} \varphi_{0}\left(e^{-h} T(\nabla)\left(\delta_{i}(h)\right) \delta_{i}(h)\right),
\end{aligned}
$$

where

$$
T(s)=\frac{1}{2} \frac{e^{-s}-1}{-s}+G(s)=\frac{-2 s+e^{s}-e^{-s}(2 s+3)+2}{4 s^{2}} .
$$

The Taylor expansion of $T$ at 0 is of the form

$$
T(s)=\frac{1}{4}-\frac{s}{12}+\frac{s^{2}}{16}-\frac{s^{3}}{80}+\frac{s^{4}}{288}-\frac{s^{5}}{2016}+O\left(s^{6}\right) .
$$

We have $\lim _{s \rightarrow \infty} T(s)=\infty, \lim _{s \rightarrow-\infty} T(s)=\infty$. Moreover, this function is non-negative as its absolute minimum is approximately 0.218207 which is attained around $s=0.812394$. Here is the graph of this function:

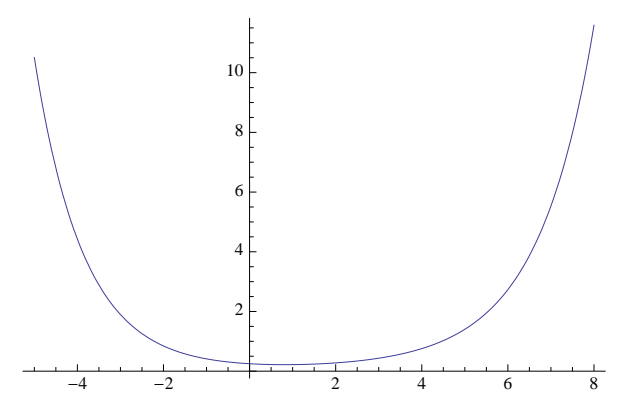

Graph of the function $T$.

Since $T$ is a non-negative function and $\delta_{i}(h)^{*}=-\delta_{i}(h)$ for $i=1, \ldots, 4$, we have

$$
\varphi_{0}\left(e^{-h} T(\nabla)\left(\delta_{i}(h)\right) \delta_{i}(h)\right)=-\varphi_{0}\left(e^{-h} T(\nabla)\left(\delta_{i}(h)\right) \delta_{i}(h)^{*}\right) \leq 0 .
$$

In the last inequality we have used the fact that $\nabla$ is a selfadjoint operator with respect to the inner product

$$
(a, b)=\varphi_{0}\left(e^{-h} b^{*} a\right), \quad a, b \in C\left(\mathbb{T}_{\theta}^{4}\right) .
$$


Also, faithfulness of $\varphi_{0}$ implies that for each $i=1, \ldots, 4$,

$$
\varphi_{0}\left(e^{-h} T(\nabla)\left(\delta_{i}(h)\right) \delta_{i}(h)\right)=0
$$

if and only if $\delta_{i}(h)=0$. Thus, it follows from (5.3) that

$$
\varphi_{0}(R)=\sum_{i=1}^{4} \varphi_{0}\left(e^{-h} T(\nabla)\left(\delta_{i}(h)\right) \delta_{i}(h)\right) \leq 0,
$$

and the equality happens if and only $\delta_{i}(h)=0$ for all $i=1, \ldots, 4$, which holds if and only if $h$ is a constant.

\section{References}

[1] N. Berline, E. Getzler, M. Vergne, Heat kernels and Dirac operators, Springer-Verlag, Berlin, 2004. Zbl 1037.58015 MR 2273508

[2] T. A. Bhuyain, M. Marcolli, The Ricci flow on noncommutative two-tori, Lett. Math. Phys. 101 (2012), no. 2, 173-194. Zbl 1261.53063 MR 2947960

[3] T. P. Branson, B. Ørsted, Conformal indices of Riemannian manifolds, Compositio Math. 60 (1986), no. 3, 261-293. Zbl 0608.58039 MR 869104

[4] P. B. Cohen, A. Connes, Conformal geometry of the irrational rotation algebra, Preprint MPI (92-93).

[5] A. Connes, $C^{*}$-algèbres et géométrie différentielle, C. R. Acad. Sc. Paris, t. 290, Série A, (1980) 599-604. Zbl 0433.46057 MR 572645

[6] A. Connes, Noncommutative differential geometry, Inst. Hautes Études Sci. Publ. Math. 62 (1985) 257-360. Zbl 0592.46056 MR 823176

[7] A. Connes, The action functional in noncommutative geometry, Comm. Math. Phys. 117 (1988) no. 4, 673-683. MR 953826

[8] A. Connes, Noncommutative geometry, Academic Press, 1994. Zbl 0818.46076 MR 1303779

[9] A. Connes, M. Marcolli, Noncommutative Geometry, Quantum Fields and Motives, American Mathematical Society Colloquium Publications, 55, 2008. Zbl 1209.58007 MR 2371808

[10] A. Connes, H. Moscovici, The local index formula in noncommutative geometry, Geom. Funct. Anal. 5 (1995) no. 2, 174-243. Zbl 0960.46048 MR 1334867

[11] A. Connes, H. Moscovici, Modular curvature for noncommutative two-tori, J. Amer. Math. Soc. 27 (2014), no. 3, 639-684. Zbl 06346499 MR 3194491

[12] A. Connes, P. Tretkoff, The Gauss-Bonnet theorem for the noncommutative two torus, Noncommutative geometry, arithmetic, and related topics, 141-158, Johns Hopkins Univ. Press, Baltimore, MD, 2011. Zbl 1251.46037 MR 2907006 
[13] L. Dabrowski, A. Sitarz, Curved noncommutative torus and Gauss-Bonnet, J. Math. Phys. 54 (2013) 013518 11pp. Zbl 1285.58014 MR 3059906

[14] L. Dabrowski, A. Sitarz, Asymmetric noncommutative torus, arXiv:1406.4645, 2014.

[15] F. Fathizadeh, M. Khalkhali, The Gauss-Bonnet theorem for noncommutative two tori with a general conformal structure, J. Noncommut. Geom. 6 (2012), no. 3, 457-480. Zbl 1256.58002 MR 2956317

[16] F. Fathizadeh, M. Khalkhali, Scalar curvature for the noncommutative two torus, J. Noncommut. Geom. 7 (2013), no. 4, 1145-1183. Zbl 1295.46053 MR 3148618

[17] F. Fathizadeh, M. Khalkhali, Weyl's Law and Connes' Trace Theorem for Noncommutative Two Tori, Lett. Math. Phys. 103 (2013), no. 1, 1-18. Zbl 1272.46055 MR 3004814

[18] F. Fathizadeh, M. W. Wong, Noncommutative residues for pseudo-differential operators on the noncommutative two-torus, Journal of Pseudo-Differential Operators and Applications, 2 (2011), no. 3, 289-302. Zbl 1266.58010 MR 2831659

[19] P. Gilkey, Invariance theory, the heat equation, and the Atiyah-Singer index theorem, Mathematics Lecture Series, 11. Publish or Perish, Inc., Wilmington, DE, 1984. Zbl 0565.58035 MR 783634

[20] V. Guillemin, A new proof of Weyl's formula on the asymptotic distribution of eigenvalues, Adv. in Math. 55 (1985), no. 2, 131-160. Zbl 0559.58025 MR 772612

[21] F. Nicola and L. Rodino, Global Pseudo-Differential Calculus on Euclidean Spaces, Birkhäuser, 2010. Zbl 1257.47002 MR 2668420

[22] M. Wodzicki, Noncommutative residue. I. Fundamentals, in $K$-theory, Arithmetic and Geometry (Moscow, 1984-1986), Lecture Notes in Mathematics 1289, Springer, 320399, 1987. Zbl 0649.58033 MR 923140

Received 02 May, 2013

F. Fathizadeh, Department of Mathematics, The University of Western Ontario, London, ON N6A 5B7, Canada

E-mail: ffathiz@uwo.ca

Current address: Department of Mathematics, Mail Code 253-37, Caltech,1200 E. California Blvd., Pasadena, CA 91125, USA

E-mail: farzadf@caltech.edu

M. Khalkhali, Department of Mathematics, The University of Western Ontario, London, ON N6A 5B7, Canada

E-mail: masoud@uwo.ca 